\title{
Equal Order Discontinuous Finite Volume Element Methods for the Stokes Problem
}

\author{
Sarvesh Kumar • Ricardo Ruiz-Baier
}

Received: 4 August 2014 / Revised: 7 November 2014 / Accepted: 26 January 2015

Published online: 6 February 2015

(C) Springer Science+Business Media New York 2015

\begin{abstract}
The aim of this paper is to develop and analyze a family of stabilized discontinuous finite volume element methods for the Stokes equations in two and three spatial dimensions. The proposed scheme is constructed using a baseline finite element approximation of velocity and pressure by discontinuous piecewise linear elements, where an interior penalty stabilization is applied. A priori error estimates are derived for the velocity and pressure in the energy norm, and convergence rates are predicted for velocity in the $L^{2}$-norm under the assumption that the source term is locally in $H^{1}$. Several numerical experiments in two and three spatial dimensions are presented to validate our theoretical findings.
\end{abstract}

Keywords Stokes equations - Discontinuous Galerkin methods - Stabilization · Finite volume element methods · Error analysis

Mathematics Subject Classification $\quad 65 \mathrm{~N} 08 \cdot 65 \mathrm{~N} 12 \cdot 76 \mathrm{D} 07 \cdot 65 \mathrm{~N} 15$

\section{Introduction}

\subsection{Scope}

Our interest is in the analysis of accurate and robust numerical methods for the discretization of the Stokes equations, which are among the simplest prototypes exhibiting some key properties and difficulties associated to the analysis and numerics of more general flow problems

\author{
S. Kumar \\ Department of Mathematics, Indian Institute of Space Science and Technology, \\ Thiruvananthapuram 695 547, Kerala, India \\ e-mail: sarvesh@iist.ac.in \\ R. Ruiz-Baier $(\varangle)$ \\ Institut des Sciences de la Terre, FGSE, Université de Lausanne, \\ Géopolis Quartier Unil-Mouline, 1015 Lausanne, Switzerland \\ e-mail: ricardo.ruizbaier@unil.ch


(as for instance, a saddle-point structure) [22]. Here we focus on discontinuous finite volume element (DFVE) methods that, by construction, retain some properties of discontinuous Galerkin (DG) and finite volume element (FVE) methods.

The typical inter-element continuity criterion, usually imposed on the finite dimensional spaces for conforming and nonconforming finite elements, is no longer required for DG methods. Other good features of DG methods include suitability for local mesh adaptation, flexibility in the definition of numerical fluxes, easiness of implementation of high order variants, natural handling of boundary conditions, improvement of mass conservation, high localizabilty and parallelizability, and so on. These have impulsed the development of an important body of bibliography concerning their numerical analysis and application for many types of flow equations (see [3] and the references therein for a review). Well-known drawbacks of DG methods are the increased computational cost associated to a larger number of degrees of freedom to achieve a fixed accuracy, and the lack of robustness with respect to stabilization parameters [6]. For Stokes flow, discontinuous approximations of velocity and pressure (usually with polynomial degrees of order $k$ and $k-1$, respectively) have been extensively studied in the literature, including different types of interior penalty (IP) methods [27], or imposing continuity of normal and tangential velocities in a weak sense [32]. Equal order approximations were introduced in the framework of local discontinuous Galerkin (LG) and IP methods in $[16,17]$, respectively. Related IP, LDG, and $h p$-methods for equal order formulations include also [42-44], and the analysis of DG methods for Stokes equations under minimal regularity, recently developed in [4].

On the other hand, FVE methods can be considered as Petrov-Galerkin schemes where the trial finite dimensional space is associated with a primal grid and the test space is constructed on a so-called dual grid (see the early work [12] and the recent review [34]). The fundamental properties of these hybrid methods, which consist in local conservativity of fluxes (as in classical finite volumes) and suitability for rigorous error analysis (as in finite elements), have turned FVE discretizations quite appealing in the numerical approximation of conservation laws and fluid mechanics in a broad sense. Some advantages of FVE with respect to classical FE methods consist in that approximate solutions are constructed with piecewise constant elements on the dual grids, and so the computational burden is typically lower, but still achieving the same convergence rates as the underlying FE discretization. The disadvantage is that additional regularity is required on the exact solution or the given data in order to derive optimal $L^{2}$-estimates. For instance, for non-homogeneous elliptic problems, the analysis of optimal $L^{2}$-convergence requires either an exact solution belonging to $H^{3}$, or a source term globally in $H^{1}$ (see e.g. [20]). A variety of FVE discretizations for Stokes problems are available from the literature, including lumped approximations, covolume methods, stabilized formulations, hybrid discretizations, pressure projection methods, and many others $[13,14$, $18,19,21,33,37,39]$.

The main goal in DFVE methods is to keep desirable features of both FVE and DG methods, as proposed in [46,47]. In this method, piecewise constants defined on a so-called diamond dual grid are employed as test functions for the velocity. Then, the momentum equation is tested against these dual-based functions, yielding a pure finite volume (FV) formulation. Properties of a lump operator transforming piecewise linear functions defined on the primal triangular (or tetrahedral) mesh into piecewise constant functions associated to the diamond mesh, allow us to recast the resulting FV formulation almost entirely back on the primal mesh. Only the right hand side of the discrete momentum equation will eventually include terms defined on the diamond mesh, and so the dual mesh will be employed only in the assembly of the datum. Further advantages of DFVE methods over classical FVE discretizations reside mainly on local conservation properties at the control volume level, 
smaller control volumes (less than half the size of control volumes typically employed in existing FVE and FV methods), and the liberty to choose tailored numerical fluxes as done in FV methods. DFVE approximations of elliptic problems have been analyzed in $[9,31,35$, $36,45]$, whereas their application to flow problems has been analyzed in only a few recent papers $[19,48,49]$. These studies deal with two-dimensional formulations of the Stokes (and, respectively, generalized Stokes) equations using discontinuous $\mathbb{P}_{1}-\mathbb{P}_{0}$ approximations of velocity and pressure. We also mention the related vertex-centered DG scheme studied in [8], and an equal-order staggered DG method recently introduced for Stokes problems in [29] (see also [15]).

The present work differs from the contributions mentioned above in that here we introduce a fully discontinuous FVE formulation of the Stokes problem in its primal formulation and constructed on the basis of an equal order approximation of velocity and pressure, where our analysis and implementation are performed for two- and three-dimensional domains. Classical tools of duality and energy methods are employed in the derivation of convergence rates for the proposed formulation. We stress that our next goal is to study discontinuous FVE approximations of coupled flow and transport problems modeling the sedimentation of suspensions (see e.g. [11,30,41]) where the advantages of DFVE methods become more apparent; and we believe the rigorous analysis of an equal order DFVE formulation for the Stokes equations is a required intermediate step.

We have arranged the contents of this paper as follows. The remainder of this section recalls some standard notation, contains a brief summary of the velocity-pressure formulation for the Stokes equations in strong and variational form, and it states a solvability result. Section 2 deals with the detailed derivation of our DFVE scheme, and collects some preliminary results. The stability, solvability, and convergence analysis of the proposed method are established in Sect. 3, and we show in Sect. 4 several numerical examples confirming the derived convergence rates. We close with some conclusions and outlook in Sect. 5.

\subsection{Preliminaries}

Let $\Omega \subset \mathbb{R}^{d}, d=2,3$, be a bounded domain with polygonal boundary $\Gamma=\partial \Omega$ and outward unit normal vector $\boldsymbol{n}$ to $\Gamma$. Standard notation will be assumed for Lebesgue spaces $L^{p}(\Omega)$, $1 \leq p \leq \infty, L_{0}^{2}(\Omega)=\left\{q \in L^{2}(\Omega): \int_{\Omega} q \mathrm{~d} x=0\right\}$ and Sobolev functional spaces $H^{m}(\Omega)$. We also denote $\mathbf{H}^{m}(\Omega)=H^{m}(\Omega)^{d}, \mathbf{H}_{0}^{1}(\Omega):=\left\{\boldsymbol{v} \in \mathbf{H}^{1}(\Omega):\left.\boldsymbol{v}\right|_{\Gamma}=0\right\}$, and in general $\mathbf{H}$ will stand for the corresponding vectorial counterpart of the scalar space $H$. For a subset $S \subset \Omega,(\cdot, \cdot)_{S}$ denotes the $L^{2}(S)$-inner product, and we will simply write $(\cdot, \cdot)$ when $S=\Omega$ or when no confusion can arise. As usual, we call $\mathbb{P}_{r}(S)$ the space of polynomial functions of degree $s \leq r$ defined on $S$.

\subsection{Governing Equations}

Let us consider the classical Stokes equations in their primal formulation describing the steady motion of an incompressible fluid

$$
\begin{aligned}
-\operatorname{div}(v \boldsymbol{\varepsilon}(\boldsymbol{u})-p \mathbf{I}) & =\boldsymbol{f} & & \text { in } \Omega, \\
\operatorname{div} \boldsymbol{u} & =0 & & \text { in } \Omega, \\
\boldsymbol{u} & =\mathbf{0} & & \text { on } \Gamma,
\end{aligned}
$$

where the unknowns are the fluid velocity $\boldsymbol{u}$ and the pressure field $p$. Here $v \boldsymbol{\varepsilon}(\boldsymbol{u})-p \mathbf{I}$ is the Cauchy stress tensor, $\boldsymbol{\varepsilon}(\boldsymbol{u})=\frac{1}{2}\left(\nabla \boldsymbol{u}+\nabla \boldsymbol{u}^{\mathrm{t}}\right)=\frac{1}{2}\left(\frac{\partial u_{i}}{\partial x_{j}}+\frac{\partial u_{j}}{\partial x_{i}}\right)=\boldsymbol{\varepsilon}_{i j}(\boldsymbol{u}), 1 \leq i, j \leq$ 
$d$ is the infinitesimal rate of strain, $v>0$ is a constant viscosity and $f \in \mathbf{L}^{2}(\Omega)$ is a vector function specifying applied body force. Multiplication by adequate test functions and integration by parts over $\Omega$ yields the following weak formulation to (1.1)-(1.3): Find $(\boldsymbol{u}, p) \in \mathbf{H}_{0}^{1}(\Omega) \times L_{0}^{2}(\Omega)$ such that

$$
\begin{aligned}
a(\boldsymbol{u}, \boldsymbol{v})-b(\boldsymbol{v}, p) & =F(\boldsymbol{v}) \quad \forall \boldsymbol{v} \in \mathbf{H}_{0}^{1}(\Omega), \\
b(\boldsymbol{u}, q) & =0 \quad \forall q \in L^{2}(\Omega),
\end{aligned}
$$

where the involved bilinear forms $a(\cdot, \cdot): \mathbf{H}_{0}^{1}(\Omega) \times \mathbf{H}_{0}^{1}(\Omega) \rightarrow \mathbb{R}$ and $b(\cdot, \cdot): \mathbf{H}_{0}^{1}(\Omega)$ $\times L_{0}^{2}(\Omega) \rightarrow \mathbb{R}$ and the linear functional $F(\cdot): \mathbf{H}_{0}^{1}(\Omega) \rightarrow \mathbb{R}$, are defined as usual:

$$
a(\boldsymbol{u}, \boldsymbol{v}):=\int_{\Omega} v \boldsymbol{\varepsilon}(\boldsymbol{u}): \boldsymbol{\varepsilon}(\boldsymbol{v}) \mathrm{d} x, \quad b(\boldsymbol{v}, q):=\int_{\Omega} q \operatorname{div} \boldsymbol{v} \mathrm{d} x, \quad F(\boldsymbol{v}):=(\boldsymbol{f}, \boldsymbol{v}),
$$

for all $\boldsymbol{u}, \boldsymbol{v} \in \mathbf{H}_{0}^{1}(\Omega)$ and $q \in L_{0}^{2}(\Omega)$.

Finally, let us also recall the definition of the usual norms:

$$
\begin{aligned}
\|\boldsymbol{u}\|_{0, \Omega}^{2} & :=\sum_{i=1}^{d}\left\|u_{i}\right\|_{0, \Omega}^{2}=\sum_{i=1}^{d} \int_{\Omega}\left(u_{i}\right)^{2} \mathrm{~d} x, \quad|\boldsymbol{u}|_{1, \Omega}^{2}:=\sum_{i=1}^{d}\left|u_{i}\right|_{1, \Omega}^{2}=\sum_{i=1}^{d} \int_{\Omega}\left|\nabla u_{i}\right|^{2} \mathrm{~d} x, \\
\|\boldsymbol{u}\|_{1, \Omega}^{2} & :=|\boldsymbol{u}|_{1, \Omega}^{2}+\|\boldsymbol{u}\|_{0, \Omega}^{2}, \quad\|\boldsymbol{\varepsilon}(\boldsymbol{u})\|_{0, \Omega}^{2}:=\sum_{i, j=1}^{d}\left\|\boldsymbol{\varepsilon}_{i j}(\boldsymbol{u})\right\|_{0, \Omega}^{2}=\sum_{i, j=1}^{d} \int_{\Omega}\left(\boldsymbol{\varepsilon}_{i j}(\boldsymbol{u})\right)^{2} \mathrm{~d} x .
\end{aligned}
$$

An application of Poincaré inequality together with Korn's inequality confirms the coercivity of the bilinear form $a(\cdot, \cdot)$ in $\mathbf{H}_{0}^{1}(\Omega)$. In addition, there exist a $\zeta>0$ such that

$$
\inf _{q \in L_{0}^{2}(\Omega)} \sup _{\boldsymbol{v} \in \mathbf{H}_{0}^{1}(\Omega) \backslash\{\boldsymbol{0}\}} \frac{b(\boldsymbol{v}, q)}{\|\boldsymbol{v}\|_{1, \Omega}\|q\|_{0, \Omega}} \geq \zeta,
$$

and hence the unique solvability of problem (1.4) is ensured (see e.g. [10] for further details).

\section{Finite Volume Element Discretization}

Let $\mathcal{T}_{h}$ be a regular family of meshes of $\Omega$ formed by closed triangular (tetrahedral if $d=3$ ) elements $K$ with boundary $\partial K$ and diameter $h_{K}$, and by vertices $s_{j}, j=1, \ldots, N_{h}$. Each face $\sigma$ between two neighboring elements $K$ and $L$ has diameter $h_{\sigma}$. The set of all faces in $\mathcal{T}_{h}$ is denoted by $\mathcal{E}_{h}$ and $\mathcal{E}_{h}^{\Gamma}$ is its restriction to boundary faces.

Associated to the mesh $\mathcal{T}_{h}$ with meshsize $h=\max _{K \in \mathcal{T}_{h}}\left(h_{K}\right)$, we define the following trial finite dimensional subspaces

$$
\begin{aligned}
& \mathcal{V}_{h}=\left\{\boldsymbol{v} \in \mathbf{L}^{2}(\Omega):\left.\boldsymbol{v}\right|_{K} \in \mathbb{P}_{1}(K)^{d}, \forall K \in \mathcal{T}_{h}\right\}, \\
& \mathcal{Q}_{h}=\left\{q \in L_{0}^{2}(\Omega):\left.q\right|_{K} \in \mathbb{P}_{1}(K), \forall K \in \mathcal{T}_{h}\right\},
\end{aligned}
$$

for the classical DG approximation of velocity and pressure, respectively. Let $\boldsymbol{n}_{K, \sigma}$ denote the outward vector of $K \in \mathcal{T}_{h}$ normal to $\sigma \subset \partial K$. For a scalar function $q \in L^{2}(\Omega)$ we let $\llbracket q \rrbracket_{\sigma}$ $:=\left.q\right|_{K}-\left.q\right|_{L}$ denote a scalar jump across $\sigma=\bar{K} \cap \bar{L}$ and $\llbracket q \boldsymbol{n}_{K, \sigma} \rrbracket_{\sigma}:=\left.q\right|_{\partial K} \boldsymbol{n}_{K, \sigma}-\left.q\right|_{\partial L} \boldsymbol{n}_{L, \sigma}$ denote a vector jump across $\sigma$. By $\{q\}_{\sigma}$ we denote its average value on $\sigma$. If $\sigma \in \mathcal{E}_{h}^{\Gamma}$, then we simply consider $\llbracket q \rrbracket_{\sigma}=\{q\}_{\sigma}=\left.q\right|_{\sigma}$. For $\boldsymbol{v}_{h} \in \mathcal{V}_{h}, \llbracket \boldsymbol{v}_{h} \rrbracket_{\sigma}=\left.\boldsymbol{v}_{h}\right|_{K}-\left.\boldsymbol{v}_{h}\right|_{L}$ and $\left\{\boldsymbol{v}_{h}\right\}_{\sigma}=\frac{1}{2}\left(\left.\boldsymbol{v}_{h}\right|_{K}+\left.\boldsymbol{v}_{h}\right|_{L}\right)$ denote, respectively, its jump and average across $\sigma$. 
As it is well known, the lowest equal order pair of finite elements does not fulfill the infsup condition unless suitable stabilizer terms are added in the baseline discrete formulation. We choose, in particular, a one parameter family interior penalty discretization (in terms of $\theta \in\{-1,0,1\})$ of the viscous term combined with a pressure jump stabilization, leading to the following DG formulation of the Stokes equations (see closely related methods in e.g. $[5,16,23,25,28,43,44])$ :

Find $\left(\tilde{\boldsymbol{u}}_{\mathbf{h}}, \tilde{p}_{h}\right) \in \mathcal{V}_{h} \times \mathcal{Q}_{h}$ such that

$$
\begin{aligned}
& a_{h}\left(\tilde{\boldsymbol{u}}_{\mathbf{h}}, \boldsymbol{v}_{h}\right)-b_{h}\left(\boldsymbol{v}_{h}, \tilde{p}_{h}\right)=F\left(\boldsymbol{v}_{h}\right) \quad \forall \boldsymbol{v}_{h} \in \mathcal{V}_{h}, \\
& b_{h}\left(\tilde{\boldsymbol{u}}_{\mathbf{h}}, q_{h}\right)+d_{h}\left(\tilde{p}_{h}, q_{h}\right)=G_{h}\left(q_{h}\right) \quad \forall q_{h} \in \mathcal{Q}_{h},
\end{aligned}
$$

where the discrete bilinear forms $a_{h}(\cdot, \cdot), b_{h}(\cdot, \cdot), d_{h}(\cdot, \cdot)$ and the linear functional $G_{h}(\cdot)$ are defined as:

$$
\begin{aligned}
a_{h}\left(\boldsymbol{w}_{h}, \boldsymbol{v}_{h}\right):= & a\left(\boldsymbol{w}_{h}, \boldsymbol{v}_{h}\right)-\sum_{\sigma \in \mathcal{E}_{h}} v \int_{\sigma}\left(\left\{\boldsymbol{\varepsilon}\left(\boldsymbol{w}_{h}\right) \boldsymbol{n}\right\}_{\sigma} \cdot \llbracket \boldsymbol{v}_{h} \rrbracket_{\sigma}+\theta\left\{\boldsymbol{\varepsilon}\left(\boldsymbol{v}_{h}\right) \boldsymbol{n}\right\}_{\sigma} \cdot \llbracket \boldsymbol{w}_{h} \rrbracket_{\sigma}\right) \mathrm{d} s, \\
& +\sum_{\sigma \in \mathcal{E}_{h}} \int_{\sigma} \frac{\alpha_{c} v}{h_{\sigma}^{\beta}} \llbracket \boldsymbol{w}_{h} \rrbracket_{\sigma} \cdot \llbracket \boldsymbol{v}_{h} \rrbracket_{\sigma} \mathrm{d} s, \\
b_{h}\left(\boldsymbol{v}_{h}, q_{h}\right):= & b\left(\boldsymbol{v}_{h}, q_{h}\right)+\sum_{\sigma \in \mathcal{E}_{h}} \int_{\sigma}\left\{q_{h} \boldsymbol{n}\right\}_{\sigma} \cdot \llbracket \boldsymbol{v}_{h} \rrbracket_{\sigma} \mathrm{d} s, \\
d_{h}\left(r_{h}, q_{h}\right):= & \sum_{K \in \mathcal{T}_{h}} \frac{\alpha_{d}}{v} h_{K}^{2}\left(\nabla r_{h}, \nabla q_{h}\right)_{K}+\sum_{\sigma \in \mathcal{E}_{h}} \int_{\sigma} \frac{\alpha_{e}}{v} h_{\sigma} \llbracket r_{h} \rrbracket_{\sigma} \llbracket q_{h} \rrbracket_{\sigma} \mathrm{d} s, \\
G_{h}\left(q_{h}\right):= & \sum_{K \in \mathcal{T}_{h}} \alpha_{d} h_{K}^{2}\left(\boldsymbol{f}, \nabla q_{h}\right)_{K},
\end{aligned}
$$

for all $\boldsymbol{w}_{h}, \boldsymbol{v}_{h} \in \mathcal{V}_{h}, q_{h}, r_{h} \in \mathcal{Q}_{h}$, where $\theta \in\{-1,0,1\}$ is the penalty parameter and $\alpha_{i}>0, \beta>0, i \in\{c, d, e\}$ are stabilization parameters (independent of $h$ ), to be specified later. Throughout the paper, we will denote by $C$, with or without subscripts, a generic positive constant that may depend on the domain $\Omega$, but not on the mesh parameter $h$.

In addition, we define the more regular space for the discrete velocity $\mathbf{V}(h):=\mathcal{V}_{h}+$ $\left(\mathbf{H}^{2}(\Omega) \bigcap \mathbf{H}_{0}^{1}(\Omega)\right)$. Since the overall discretization is nonconforming, we also define the following discrete norms on $\mathbf{V}(h)$ and $\mathcal{Q}_{h}$ to be utilized in the forthcoming analysis [5,23,30]:

$$
\begin{aligned}
\left\|\boldsymbol{v}_{h}\right\|_{h}^{2} & :=\sum_{K \in \mathcal{T}_{h}}\left|\boldsymbol{v}_{h}\right|_{1, K}^{2}+\sum_{\sigma \in \mathcal{E}_{h}} h_{\sigma}^{-\beta}\left\|\llbracket \boldsymbol{v}_{h} \rrbracket_{\sigma}\right\|_{0, \sigma}^{2}, \quad\left\|\boldsymbol{v}_{h}\right\|^{2}:=\left\|\boldsymbol{v}_{h}\right\|_{h}^{2}+\sum_{K \in \mathcal{T}_{h}} h_{K}^{2}\left|\boldsymbol{v}_{h}\right|_{2, K}^{2}, \\
\left\|q_{h}\right\|_{h}^{2} & :=\sum_{K \in \mathcal{T}_{h}}\left\|q_{h}\right\|_{0, K}^{2}+h_{K}^{2}\left|q_{h}\right|_{1, K}^{2}+\sum_{\sigma \in \mathcal{E}_{h}} h_{\sigma}\left\|\llbracket q_{h} \rrbracket_{\sigma}\right\|_{0, \sigma}^{2} .
\end{aligned}
$$

The standard inverse inequality implies that there exists $C>0$ such that

$$
\left\|\boldsymbol{v}_{h}\right\| \leq C\left\|\boldsymbol{v}_{h}\right\|_{h} \quad \forall \boldsymbol{v}_{h} \in \mathcal{V}_{h} .
$$

We will also make use of the following well established trace inequalities (cf. [1, Th.3.10])

$$
\begin{aligned}
\|\boldsymbol{v}\|_{0, \sigma}^{2} \leq C\left(h_{K}^{-1}\|\boldsymbol{v}\|_{0, K}^{2}+h_{K}|\boldsymbol{v}|_{1, K}^{2}\right) & \forall \boldsymbol{v} \in \mathbf{H}^{1}(K), \\
\left\|(\nabla \boldsymbol{v}) \boldsymbol{n}_{K, \sigma}\right\|_{0, \sigma}^{2} \leq C\left(h_{K}^{-1}|\boldsymbol{v}|_{1, K}^{2}+h_{K}|\boldsymbol{v}|_{2, K}^{2}\right) & \forall \boldsymbol{v} \in \mathbf{H}^{2}(K),
\end{aligned}
$$

for $\sigma \subset \partial K$, where $C>0$ depends also on the minimum angle of $K \in \mathcal{T}_{h}$. 

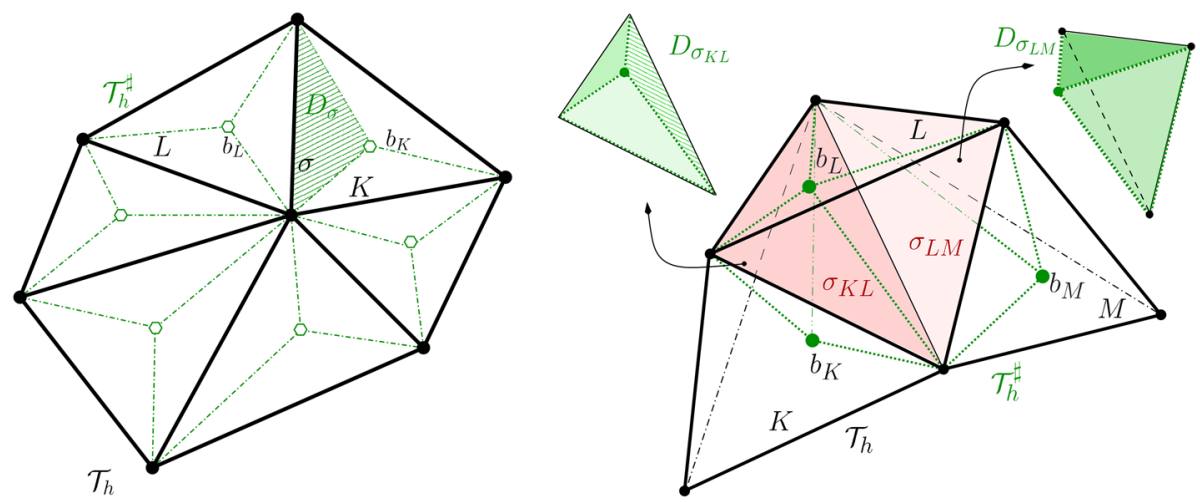

Fig. 1 Left: Triangular elements in $\mathcal{T}_{h}$ (solid lines) and diamonds in $\mathcal{T}_{h}^{\sharp}$ (dashed-dotted lines). Right: Sketch of tetrahedrons in $\mathcal{T}_{h}$ and diamonds of $\mathcal{T}_{h}^{\sharp}$ (dashed lines)

We next introduce a so-called diamond mesh $\mathcal{T}_{h}^{\sharp}$ consisting of diamonds $D_{\sigma}$ generated by barycentric subdivision, i.e., each diamond $D_{\sigma} \in \mathcal{T}_{h}^{\sharp}$ is associated to the face $\sigma \in \mathcal{E}_{h}$ and constructed by joining the barycenters $b_{K}$ and $b_{L}$ of the elements $K$ and $L$ sharing the interior face $\sigma$, with the vertices of $\sigma$ (see Fig. 1).

Linked to the diamond mesh $\mathcal{T}_{h}^{\sharp}$, let

$$
\mathcal{V}_{h}^{\sharp}=\left\{\boldsymbol{v} \in \mathbf{L}^{2}(\Omega):\left.\boldsymbol{v}\right|_{D_{\sigma}} \in \mathbb{P}_{0}\left(D_{\sigma}\right)^{d}, \forall D_{\sigma} \in \mathcal{T}_{h}^{\sharp},\left.\boldsymbol{v}\right|_{D_{\sigma}}=0 \text { if } \sigma \in \mathcal{E}_{h}^{\Gamma}\right\},
$$

be a test space for the velocity field. For connecting trial and test spaces for the FVE approximation of velocity, we define the transfer operator $\gamma: \mathbf{V}(h) \longrightarrow \mathcal{V}_{h}^{\sharp}$ in the following manner:

$$
\left.\gamma \boldsymbol{v}\right|_{D_{\sigma}}=\left.\frac{1}{h_{\sigma}} \int_{\sigma} \boldsymbol{v}\right|_{D_{\sigma}} \mathrm{d} s, \quad D_{\sigma} \in \mathcal{T}_{h}^{\sharp} .
$$

Some of the relations satisfied by this map are collected in the following lemma.

Lemma 1 (See [30]) For $\boldsymbol{v}_{h} \in \mathcal{V}_{h}$ we have

$$
\begin{aligned}
\int_{\sigma}\left(\boldsymbol{v}_{h}-\gamma \boldsymbol{v}_{h}\right) \mathrm{d} s & =0, \\
\int_{\sigma} \llbracket \boldsymbol{v}_{h}-\gamma \boldsymbol{v}_{h} \rrbracket_{\sigma} \mathrm{d} s & =0, \\
\int_{K}\left(\boldsymbol{v}_{h}-\gamma \boldsymbol{v}_{h}\right) \mathrm{d} x & =0, \\
\left\|\boldsymbol{v}_{h}-\gamma \boldsymbol{v}_{h}\right\|_{0, K} & \leq C h_{K}\left\|\boldsymbol{v}_{h}\right\|_{1, K} .
\end{aligned}
$$

Let us now multiply (1.1) and (1.2) by $\gamma \boldsymbol{v}_{h} \in \mathcal{V}_{h}^{\sharp}$ and $q_{h} \in \mathcal{Q}_{h}$, respectively. Using Gauss divergence theorem on the diamond element $D_{\sigma}$, and adding over all diamond elements, we obtain 

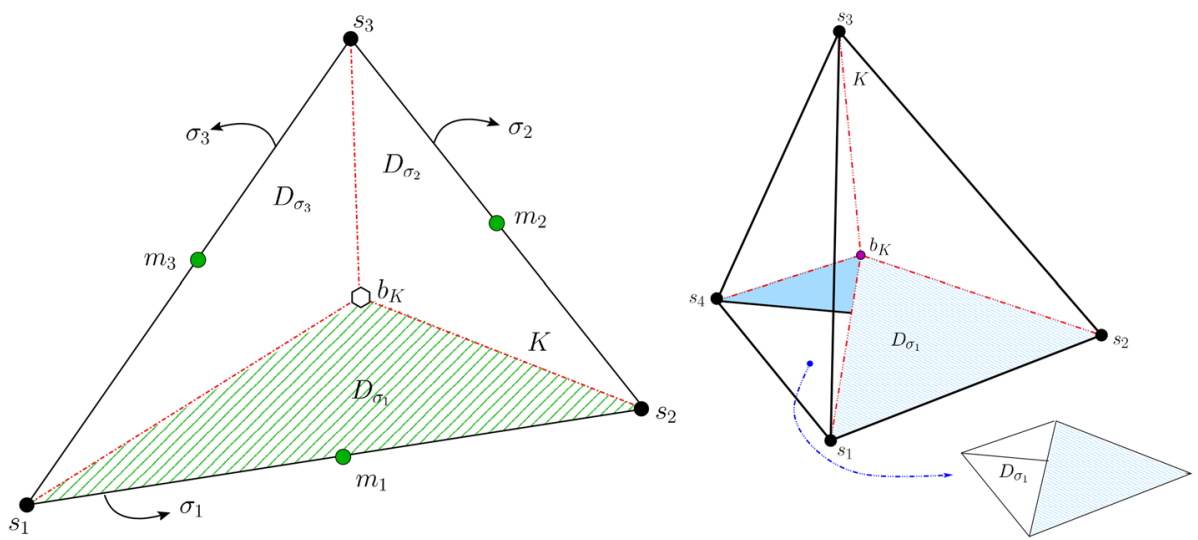

Fig. 2 Left: Triangular element $K \in \mathcal{T}_{h}$ (solid lines) and three diamonds $D_{\sigma_{j}}$ (dashed lines). Right: Threedimensional counterpart

$$
\begin{aligned}
& -\sum_{D_{\sigma} \in \mathcal{T}_{h}^{\sharp}} v \int_{\partial D_{\sigma}} \boldsymbol{\varepsilon}(\boldsymbol{u}) \boldsymbol{n} \cdot \gamma \boldsymbol{v}_{h} \mathrm{~d} s+\sum_{D_{\sigma} \in \mathcal{T}_{h}^{\sharp}} \int_{\partial D_{\sigma}} p \boldsymbol{n} \cdot \gamma \boldsymbol{v}_{h} \mathrm{~d} s=\left(\boldsymbol{f}, \gamma \boldsymbol{v}_{h}\right) \quad \forall \boldsymbol{v}_{h} \in \mathcal{V}_{h}, \\
& \sum_{K \in \mathcal{T}_{h}} \int_{K} q_{h} \operatorname{div} \boldsymbol{u} \mathrm{d} x=0 \quad \forall q_{h} \in \mathcal{Q}_{h} .
\end{aligned}
$$

Let $D_{\sigma_{j}} \in \mathcal{T}_{h}^{\sharp}(j=1, \ldots, d+1)$ be the $d+1$ sub-elements (triangles if $d=2$, or tetrahedra if $d=3$ ) formed by barycentric subdivision of a generic element $K \in \mathcal{T}_{h}$, see Fig. 2. Then, for $\gamma \boldsymbol{v}_{h} \in \mathcal{V}_{h}^{\sharp}$, we can write

$$
\begin{aligned}
& \sum_{D_{\sigma} \in \mathcal{T}_{h}^{\sharp}} v \int_{\partial D_{\sigma}} \boldsymbol{\varepsilon}(\boldsymbol{u}) \boldsymbol{n} \cdot \gamma \boldsymbol{v}_{h} \mathrm{~d} s \\
& =\sum_{K \in \mathcal{T}_{h}} \sum_{j=1}^{d+1} v \int_{\partial D_{\sigma_{j}}} \boldsymbol{\varepsilon}(\boldsymbol{u}) \boldsymbol{n} \cdot \gamma \boldsymbol{v}_{h} \mathrm{~d} s \\
& =\sum_{K \in \mathcal{T}_{h}} \sum_{j=1}^{d+1} v \int_{s_{j+1} b_{K} s_{j}} \boldsymbol{\varepsilon}(\boldsymbol{u}) \boldsymbol{n} \cdot \gamma \boldsymbol{v}_{h} \mathrm{~d} s+\sum_{K \in \mathcal{T}_{h}} v \int_{\partial K} \boldsymbol{\varepsilon}(\boldsymbol{u}) \boldsymbol{n} \cdot \gamma \boldsymbol{v}_{h} \mathrm{~d} s,
\end{aligned}
$$

where $s_{d+2}=s_{1}$.

On the other hand, notice that for any $k, l, m, n \in \mathbb{R}$, one can write the following relation

$$
k m-l n=\frac{1}{2}(k+l)(m-n)+\frac{1}{2}(k-l)(m+n) .
$$

Applying the identity (2.10) to a product of a jump and an average, and elementary computations, yields

$$
\sum_{K \mathcal{T}_{h}} \int_{\partial K} q \boldsymbol{v} \cdot \boldsymbol{n} \mathrm{d} s=\sum_{\sigma \in \mathcal{E}_{h}^{i n t}} \int_{\sigma} \llbracket q \boldsymbol{n} \rrbracket_{\sigma} \cdot\{\boldsymbol{v}\}_{\sigma} \mathrm{d} s+\sum_{\sigma \in \mathcal{E}_{h}} \int_{\sigma}\{q \boldsymbol{n}\}_{\sigma} \cdot \llbracket \boldsymbol{v} \rrbracket_{\sigma} \mathrm{d} s .
$$


Now, owing to (2.9), (2.11), and the fact that the exact solutions (u,p) of (1.1)-(1.3) satisfy $\llbracket \epsilon(\boldsymbol{u}) \boldsymbol{n} \rrbracket_{\sigma}=\mathbf{0}$ and $\llbracket p \boldsymbol{n} \rrbracket_{\sigma}=\mathbf{0}$ on every $\sigma \in \mathcal{E}_{h}^{i n t}$, we can deduce that a stabilized DFVE counterpart of (2.1)-(2.2) reads:

Find $\left(\boldsymbol{u}_{h}, p_{h}\right) \in \mathcal{V}_{h} \times \mathcal{Q}_{h}$ such that

$$
\begin{aligned}
A_{h}\left(\boldsymbol{u}_{h}, \boldsymbol{v}_{h}\right)+C_{h}\left(\boldsymbol{v}_{h}, p_{h}\right) & =F\left(\gamma \boldsymbol{v}_{h}\right) \quad \forall \boldsymbol{v}_{h} \in \mathcal{V}_{h}, \\
B_{h}\left(\boldsymbol{u}_{h}, q_{h}\right)+d_{h}\left(p_{h}, q_{h}\right) & =G_{h}\left(q_{h}\right) \quad \forall q_{h} \in \mathcal{Q}_{h},
\end{aligned}
$$

which can be rewritten in the form:

Find $\left(\boldsymbol{u}_{h}, p_{h}\right) \in \mathcal{V}_{h} \times \mathcal{Q}_{h}$ such that

$$
\mathcal{A}\left(\left(\boldsymbol{u}_{h}, p_{h}\right) ;\left(\boldsymbol{v}_{h}, q_{h}\right)\right)=F\left(\gamma \boldsymbol{v}_{h}\right)+G_{h}\left(q_{h}\right) \quad \forall\left(\boldsymbol{v}_{h}, q_{h}\right) \in \mathcal{V}_{h} \times \mathcal{Q}_{h},
$$

where the quadrilinear form is defined as: $\mathcal{A}\left(\left(\boldsymbol{u}_{h}, p_{h}\right) ;\left(\boldsymbol{v}_{h}, q_{h}\right)\right):=A_{h}\left(\boldsymbol{u}_{h}, v_{h}\right)+$ $C_{h}\left(\boldsymbol{v}_{h}, p_{h}\right)+B_{h}\left(\boldsymbol{u}_{h}, q_{h}\right)+d_{h}\left(p_{h}, q_{h}\right)$, and its components read

$$
\begin{aligned}
A_{h}\left(\boldsymbol{u}_{h}, \boldsymbol{v}_{h}\right):=- & \sum_{K \in \mathcal{T}_{h}} \sum_{j=1}^{d+1} v \int_{s_{j+1} b_{K} s_{j}} \boldsymbol{\varepsilon}\left(\boldsymbol{u}_{h}\right) \boldsymbol{n} \cdot \gamma \boldsymbol{v}_{h} \mathrm{~d} s-\sum_{\sigma \in \mathcal{E}_{h}} v \int_{\sigma}\left\{\boldsymbol{\varepsilon}\left(\boldsymbol{u}_{h}\right) \boldsymbol{n}\right\}_{\sigma} \cdot \llbracket \gamma \boldsymbol{v}_{h} \rrbracket_{\sigma} \mathrm{d} s \\
& -\theta \sum_{\sigma \in \mathcal{E}_{h}} v \int_{\sigma}\left\{\boldsymbol{\varepsilon}\left(\boldsymbol{v}_{h}\right) \boldsymbol{n}\right\}_{\sigma} \cdot \llbracket \gamma \boldsymbol{u}_{h} \rrbracket_{\sigma} \mathrm{d} s+\sum_{\sigma \in \mathcal{E}_{h}} \int_{\sigma} \frac{\alpha_{c} v}{h_{\sigma}^{\beta} \llbracket \boldsymbol{u}_{h} \rrbracket_{\sigma} \cdot \llbracket \boldsymbol{v}_{h} \rrbracket_{\sigma} \mathrm{d} s,} \\
B_{h}\left(\boldsymbol{v}_{h}, q_{h}\right):= & b\left(\boldsymbol{v}_{h}, q_{h}\right)-\sum_{\sigma \in \mathcal{E}_{h}} \int_{\sigma}\left\{q_{h} \boldsymbol{n}\right\}_{\sigma} \cdot \llbracket \gamma \boldsymbol{v}_{h} \rrbracket_{\sigma} \mathrm{d} s, \\
C_{h}\left(\boldsymbol{v}_{h}, q_{h}\right):= & \sum_{K \in \mathcal{T}_{h}} \sum_{j=1}^{d+1} \int_{s_{j+1} b_{K} s_{j}} q_{h} \gamma \boldsymbol{v}_{h} \cdot \boldsymbol{n} \mathrm{d} s+\sum_{\sigma \in \mathcal{E}_{h}} \int_{\sigma}\left\{q_{h} \boldsymbol{n}\right\}_{\sigma} \cdot \llbracket \gamma \boldsymbol{v}_{h} \rrbracket_{\sigma} \mathrm{d} s \\
& -\sum_{\sigma \in \mathcal{E}_{h}} \int_{\sigma}\left(\llbracket \boldsymbol{v}_{h}-\gamma \boldsymbol{v}_{h} \rrbracket_{\sigma} \cdot\left\{q_{h} \boldsymbol{n}\right\}_{\sigma}+\llbracket q_{h} \rrbracket_{\sigma} \cdot\left\{\left(\boldsymbol{v}_{h}-\gamma \boldsymbol{v}_{h}\right) \boldsymbol{n}\right\}_{\sigma}\right) \mathrm{d} s,
\end{aligned}
$$

for all $\boldsymbol{u}_{h}, \boldsymbol{v}_{h} \in \mathcal{V}_{h}, q_{h} \in \mathcal{Q}_{h}$.

Remark 1 We stress that the cases $\theta=1, \theta=0$ and $\theta=-1$ lead respectively to the symmetric, incomplete, and non-symmetric interior penalty formulations (SIPG, IIPG and NIPG, respectively) in the context of discontinuous Galerkin finite element methods. Some advantages and disadvantages of these methods with respect to each other are discussed in e.g. [26] (see also [3] and the references given there).

In order to show consistency of the DFVE scheme (2.14) with respect to the weak formulation (1.4), we first state the following lemma (a proof can be found in [48]).

Lemma 2 For all $(\boldsymbol{v}, q) \in \mathbf{V}(h) \times L_{0}^{2}(\Omega)$, we have

$$
\begin{aligned}
\sum_{K \in \mathcal{T}_{h}} \sum_{j=1}^{d+1} \int_{s_{j+1} b_{K} s_{j}} q \gamma \boldsymbol{v} \cdot \boldsymbol{n} \mathrm{d} s= & -\sum_{K \in \mathcal{T}_{h}} \int_{K} q \operatorname{div} \boldsymbol{v} \mathrm{d} x+\sum_{K \in \mathcal{T}_{h}} \int_{\partial K}(\boldsymbol{v}-\gamma \boldsymbol{v}) \cdot \boldsymbol{n} q \mathrm{~d} s \\
& +\sum_{K \in \mathcal{T}_{h}} \int_{K}(\boldsymbol{v}-\gamma \boldsymbol{v}) \cdot \nabla q \mathrm{~d} x .
\end{aligned}
$$


Now, according to (2.11) we can assert that

$$
\sum_{K \in \mathcal{T}_{h}} \int_{\partial K}(\boldsymbol{v}-\gamma \boldsymbol{v}) \cdot \boldsymbol{n} q \mathrm{~d} s=\sum_{\sigma \in \mathcal{E}_{h}} \int_{\sigma} \llbracket \boldsymbol{v}-\gamma \boldsymbol{v} \rrbracket_{\sigma} \cdot\{q \boldsymbol{n}\}_{\sigma} \mathrm{d} s+\sum_{\sigma \in \mathcal{E}_{h}} \int_{\sigma} \llbracket q \boldsymbol{n} \rrbracket_{\sigma} \cdot\{\boldsymbol{v}-\gamma \boldsymbol{v}\}_{\sigma} \mathrm{d} s .
$$

We conclude from (2.7) that

$$
\sum_{K \in \mathcal{T}_{h}} \int_{K}(\boldsymbol{v}-\gamma \boldsymbol{v}) \cdot \nabla q_{h} \mathrm{~d} x=0 \quad \forall q_{h} \in \mathcal{Q}_{h},
$$

and hence

$$
\sum_{K \in \mathcal{T}_{h}} \sum_{j=1}^{d+1} \int_{s_{j+1} b_{K} s_{j}} q \boldsymbol{v} \cdot \boldsymbol{n} \mathrm{d} s=-\sum_{K \in \mathcal{T}_{h}} \int_{K} q \operatorname{div} \boldsymbol{v} \mathrm{d} x \equiv-b(\boldsymbol{v}, q),
$$

which yields the following relation between the bilinear forms $B_{h}(\cdot, \cdot)$ and $C_{h}(\cdot, \cdot)$

$$
C_{h}\left(\boldsymbol{v}, q_{h}\right)=-B_{h}\left(\boldsymbol{v}, q_{h}\right) \quad \forall \boldsymbol{v} \in \mathbf{V}(h), q_{h} \in \mathcal{Q}_{h} .
$$

Since $d_{h}\left(p_{h}, q_{h}\right)$ appears in the formulation, the stabilized scheme (2.14) may not be consistent with the weak formulation in the usual sense. However, we can claim that the proposed stabilized scheme is asymptotically consistent.

Lemma 3 (Asymptotic consistency) Let $(\boldsymbol{u}, p) \in \mathbf{H}_{0}^{1}(\Omega) \times H^{1}(\Omega)$ and $\left(\boldsymbol{u}_{h}, p_{h}\right)$ be the solutions of (1.4) and (2.14), respectively. Then there exists a constant $C>0$ depending on the bounds for $\|p\|_{1, \Omega},\|f\|_{0, \Omega}$, and on the stabilization constants, but not on $h$, such that

$$
\mathcal{A}\left(\left(\boldsymbol{u}-\boldsymbol{u}_{h}, p-p_{h}\right) ;\left(\boldsymbol{v}_{h}, q_{h}\right)\right) \leq C h\left\|\boldsymbol{v}_{h}\right\|_{h}\left\|q_{h}\right\|_{h} .
$$

Proof Applying (2.14) and the definitions of $A_{h}(\cdot, \cdot)$ and $C_{h}(\cdot, \cdot)$ along with the fact that $\llbracket \boldsymbol{u} \rrbracket_{\sigma}=0$ and $\llbracket p \rrbracket_{\sigma}=0$, we arrive at

$$
\begin{aligned}
\mathcal{A}\left(\left(\boldsymbol{u}-\boldsymbol{u}_{h}, p-p_{h}\right) ;\left(\boldsymbol{v}_{h}, q_{h}\right)\right)= & d_{h}\left(p, q_{h}\right)-G_{h}\left(q_{h}\right) \\
& +\sum_{\sigma \in \mathcal{E}_{h}} \int_{\sigma} \llbracket \boldsymbol{v}_{h}-\gamma \boldsymbol{v}_{h} \rrbracket_{\sigma} \cdot\{p \boldsymbol{n}\}_{\sigma} \mathrm{d} s \quad \forall \boldsymbol{v}_{h} \in \mathcal{V}_{h}, q_{h} \in \mathcal{Q}_{h} .
\end{aligned}
$$

In addition, the definitions of $d_{h}, G_{h}$ and $\|\cdot\|_{h}$ together with Cauchy-Schwarz inequality, give the following bounds

$$
\begin{aligned}
\left|d_{h}\left(p, q_{h}\right)\right| & \leq C h\|p\|_{1, \Omega}\left\|q_{h}\right\|_{h}, \\
\left|G_{h}\left(q_{h}\right)\right| & \leq C h\|f\|_{0, \Omega}\left\|q_{h}\right\|_{h} .
\end{aligned}
$$

Note that the generic constant appearing in (2.17) and (2.18) may also depend on the bound for the stabilization parameter $\alpha_{d}$. To estimate the last term in the RHS of (2.16), we use once more Cauchy-Schwarz inequality to conclude that

$$
\begin{aligned}
& \left|\sum_{\sigma \in \mathcal{E}_{h}} \int_{\sigma} \llbracket \boldsymbol{v}_{h}-\gamma \boldsymbol{v}_{h} \rrbracket_{\sigma} \cdot\{p \boldsymbol{n}\}_{\sigma} \mathrm{d} s\right| \\
& \quad \leq\left(\sum_{\sigma \in \mathcal{E}_{h}} \int_{\sigma} h_{\sigma}^{-\beta} \llbracket \boldsymbol{v}_{h}-\gamma \boldsymbol{v}_{h} \mathbb{1}_{\sigma}^{2} \mathrm{~d} s\right)^{1 / 2}\left(\sum_{\sigma \in \mathcal{E}_{h}} \int_{\sigma} h_{\sigma}^{\beta}\{p \boldsymbol{n}\}_{\sigma}^{2} \mathrm{~d} s\right)^{1 / 2} .
\end{aligned}
$$


Next, the trace inequality (2.3) and the definition of the norm $\|\cdot\|_{h}$ with $\beta=3$, yields

$$
\left|\sum_{\sigma \in \mathcal{E}_{h}} \int_{\sigma} \llbracket \boldsymbol{v}_{h}-\gamma \boldsymbol{v}_{h} \rrbracket_{\sigma} \cdot\{p \boldsymbol{n}\}_{\sigma} \mathrm{d} s\right| \leq C h\|p\|_{1, \Omega}\left\|\boldsymbol{v}_{h}\right\|_{h},
$$

and the proof is completed after inserting the estimates obtained in (2.17) to (2.19) into (2.16).

\section{Stability, Solvability and Convergence Analysis}

For any $\boldsymbol{v}, \boldsymbol{w} \in \mathbf{V}(h)$, an application of Gauss divergence theorem yields the following relation (see [30] for further details)

$$
\begin{aligned}
A_{*}(\boldsymbol{v}, \boldsymbol{w})= & \sum_{K \in \mathcal{T}_{h}} v \int_{K} \boldsymbol{\varepsilon}(\boldsymbol{v}): \boldsymbol{\varepsilon}(\boldsymbol{w}) \mathrm{d} x+\sum_{K \in \mathcal{T}_{h}} v \int_{\partial K}(\gamma \boldsymbol{w}-\boldsymbol{w}) \boldsymbol{\varepsilon}(\boldsymbol{v}): \boldsymbol{n} \mathrm{d} s \\
& +\sum_{K \in \mathcal{T}_{h}} v \int_{K}(\operatorname{div} \boldsymbol{\varepsilon}(\boldsymbol{v})) \cdot(\boldsymbol{w}-\gamma \boldsymbol{w}) \mathrm{d} x,
\end{aligned}
$$

where,

$$
A_{*}(\boldsymbol{v}, \boldsymbol{w})=-\sum_{K \in \mathcal{T}_{h}} \sum_{j=1}^{d+1} v \int_{s_{j+1} b_{K} s_{j}} \boldsymbol{\varepsilon}(\boldsymbol{v}) \boldsymbol{n} \cdot \gamma \boldsymbol{w} \mathrm{d} s .
$$

Moreover, for any $\boldsymbol{v}_{h}, \boldsymbol{w}_{h} \in \mathcal{V}_{h}$, relation (2.5) and the fact that $\boldsymbol{v}_{h}$ is linear on each element, gives

$$
A_{*}\left(\boldsymbol{v}_{h}, \boldsymbol{w}_{h}\right)=\sum_{K \in \mathcal{T}_{h}} v \int_{K} \boldsymbol{\varepsilon}\left(\boldsymbol{v}_{h}\right): \boldsymbol{\varepsilon}\left(\boldsymbol{w}_{h}\right) \mathrm{d} x=a\left(\boldsymbol{v}_{h}, \boldsymbol{w}_{h}\right),
$$

and using Cauchy-Schwarz and trace inequality (2.3), we can infer that

$$
\begin{aligned}
& \sum_{\sigma \in \mathcal{E}_{h}} v \int_{\sigma}\left\{\boldsymbol{\varepsilon}\left(\boldsymbol{u}_{h}\right) \boldsymbol{n}\right\}_{\sigma} \cdot \llbracket \gamma \boldsymbol{v}_{h} \rrbracket_{\sigma} \mathrm{d} s \\
& \leq\left(\sum_{\sigma \in \mathcal{E}_{h}} h_{\sigma}^{-\beta} v \int_{\sigma} \llbracket \gamma \boldsymbol{v}_{h} \rrbracket_{\sigma}^{2} \mathrm{~d} s\right)^{1 / 2}\left(\sum_{\sigma \in \mathcal{E}_{h}} h_{\sigma}^{\beta} \int_{\sigma}\left\{\boldsymbol{\varepsilon}\left(\boldsymbol{u}_{h}\right) \boldsymbol{n}\right\}_{\sigma}^{2} \mathrm{~d} s\right)^{1 / 2} \\
& \quad \leq C\left(\sum_{\sigma \in \mathcal{E}_{h}} h_{\sigma}^{-\beta+1} \llbracket \gamma \boldsymbol{v}_{h} \mathbb{\rrbracket}_{\sigma}^{2}\right)^{1 / 2}\left(\sum_{K \in \mathcal{T}_{h}}\left\|\boldsymbol{\varepsilon}\left(u_{h}\right)\right\|_{0, K}^{2}\right)^{1 / 2} .
\end{aligned}
$$

Taking the norm of the strain $\boldsymbol{\varepsilon}(\boldsymbol{u})$ (defined in (1.5)), we can write

$$
\left(\sum_{K \in \mathcal{T}_{h}}\left\|\boldsymbol{\varepsilon}\left(\boldsymbol{u}_{h}\right)\right\|_{0, K}^{2}\right)^{1 / 2} \leq\left\|\boldsymbol{u}_{h}\right\|
$$

We then proceed to employ the definition of $\gamma$ and Cauchy-Schwarz inequality to arrive at

$$
\llbracket \gamma \boldsymbol{v}_{h} \rrbracket_{\sigma}^{2}=h_{\sigma}^{-2}\left(\int_{\sigma} \llbracket \boldsymbol{v}_{h} \rrbracket_{\sigma} \mathrm{d} s\right)^{2} \mathrm{~d} s \leq h_{\sigma}^{-2}\left(\int_{\sigma} \mathrm{d} s\right)\left(\int_{\sigma} \llbracket \boldsymbol{v}_{h} \rrbracket_{\sigma}^{2} \mathrm{~d} s\right)=h_{\sigma}^{-1} \int_{\sigma} \llbracket \boldsymbol{v}_{h} \rrbracket_{\sigma}^{2} \mathrm{~d} s,
$$


and hence,

$$
\sum_{\sigma \in \mathcal{E}_{h}} h_{\sigma}^{-\beta+1} \llbracket \gamma \boldsymbol{v}_{h} \rrbracket_{\sigma}^{2} \leq \sum_{\sigma \in \mathcal{E}_{h}} h_{\sigma}^{-\beta} \int_{\sigma} \llbracket \boldsymbol{v}_{h} \rrbracket_{\sigma}^{2} \mathrm{~d} s .
$$

Finally, putting (3.4) and (3.5) in (3.3) yields

$$
\sum_{\sigma \in \mathcal{E}_{h}} v \int_{\sigma}\left\{\boldsymbol{\varepsilon}\left(\boldsymbol{u}_{h}\right) \boldsymbol{n}\right\}_{\sigma} \cdot \llbracket \gamma \boldsymbol{v}_{h} \rrbracket_{\sigma} \mathrm{d} s \leq C\left(\sum_{\sigma \in \mathcal{E}_{h}} h_{\sigma}^{-\beta} \int_{\sigma} \llbracket \boldsymbol{v}_{h} \rrbracket_{\sigma}^{2} \mathrm{~d} s\right)^{1 / 2}\left\|\boldsymbol{u}_{h}\right\| .
$$

Next, we establish the coercivity and boundedness of the bilinear form $A_{h}(\cdot, \cdot)$.

Lemma 4 There exists $C>0$ independent of the mesh size $h$, such that these properties hold true:

$$
\begin{array}{ll}
A_{h}\left(\boldsymbol{v}_{h}, \boldsymbol{w}_{h}\right) \leq C\left\|\boldsymbol{v}_{h}\right\|\left\|\boldsymbol{w}_{h}\right\| & \forall \boldsymbol{v}_{h}, \boldsymbol{w}_{h} \in \mathbf{V}(h), \\
A_{h}\left(\boldsymbol{v}_{h}, \boldsymbol{v}_{h}\right) \geq C\left\|\boldsymbol{v}_{h}\right\|_{h}^{2} & \forall \boldsymbol{v}_{h} \in \mathcal{V}_{h} .
\end{array}
$$

Proof Let $\boldsymbol{v}_{h} \in \mathcal{V}_{h}$. Using (3.2), we can rewrite $A_{h}\left(\boldsymbol{v}_{h}, \boldsymbol{v}_{h}\right)$ as

$$
\begin{aligned}
A_{h}\left(\boldsymbol{v}_{h}, \boldsymbol{v}_{h}\right)= & a\left(\boldsymbol{v}_{h}, \boldsymbol{v}_{h}\right)-\sum_{\sigma \in \mathcal{E}_{h}} v \int_{\sigma}\left\{\boldsymbol{\varepsilon}\left(\boldsymbol{v}_{h}\right) \boldsymbol{n}\right\}_{\sigma} \cdot \llbracket \gamma \boldsymbol{v}_{h} \rrbracket_{\sigma} \mathrm{d} s \\
& -\theta \sum_{\sigma \in \mathcal{E}_{h}} v \int_{\sigma}\left\{\boldsymbol{\varepsilon}\left(\boldsymbol{v}_{h}\right) \boldsymbol{n}\right\}_{\sigma} \cdot \llbracket \gamma \boldsymbol{v}_{h} \rrbracket_{\sigma} \mathrm{d} s+\sum_{\sigma \in \mathcal{E}_{h}} \int_{\sigma} \frac{\alpha_{c} v}{h_{\sigma}^{\beta}} \llbracket \boldsymbol{v}_{h} \rrbracket_{\sigma}^{2} \mathrm{~d} s .
\end{aligned}
$$

Now, an application of (3.6) together with the discrete Korn's inequality (with constant $\alpha_{0}$ ), yields

$$
\begin{aligned}
A_{h}\left(\boldsymbol{v}_{h}, \boldsymbol{v}_{h}\right) \geq & \alpha_{0} \sum_{K \in \mathcal{T}_{h}}\left|\boldsymbol{v}_{h}\right|_{1, K}^{2}-C(1+\theta)\left\|\boldsymbol{v}_{h}\right\|_{h}\left(\sum_{\sigma \in \mathcal{E}_{h}} h_{\sigma}^{-\beta} \int_{\sigma} \llbracket \boldsymbol{v}_{h} \rrbracket_{\sigma}^{2} \mathrm{~d} s\right)^{1 / 2} \\
& +\sum_{\sigma \in \mathcal{E}_{h}} \int_{\sigma} \frac{\alpha_{c} v}{h_{\sigma}^{\beta}} \llbracket \boldsymbol{v}_{h} \mathbb{1}_{\sigma}^{2} \mathrm{~d} s,
\end{aligned}
$$

and employing Young's inequality and the definition of the mesh-dependent norm $\|\cdot\|_{h}$, we arrive at

$$
\begin{aligned}
A_{h}\left(\boldsymbol{v}_{h}, \boldsymbol{v}_{h}\right) \geq & \alpha_{0} \sum_{K \in \mathcal{T}_{h}}\left|\boldsymbol{v}_{h}\right|_{1, K}^{2}-\frac{\alpha_{0}}{2}\left\|\boldsymbol{v}_{h}\right\|_{h}^{2}-\frac{C^{2}(1+\theta)^{2}}{2 \alpha_{0}}\left(\sum_{\sigma \in \mathcal{E}_{h}} h_{\sigma}^{-\beta} \int_{\sigma} \llbracket \boldsymbol{v}_{h} \mathbb{1}_{\sigma}^{2} \mathrm{~d} s\right) \\
& +\sum_{\sigma \in \mathcal{E}_{h}} \int_{\sigma} \frac{\alpha_{c} v}{h_{\sigma}^{\beta}} \llbracket \boldsymbol{v}_{h} \mathbb{1}_{\sigma}^{2} \mathrm{~d} s \\
\geq & \frac{\alpha_{0}}{2} \sum_{K \in \mathcal{T}_{h}}\left|\boldsymbol{v}_{h}\right|_{1, K}^{2}+\left(\alpha_{c} v-\frac{C^{2}(1+\theta)^{2}}{2 \alpha_{0}}-\frac{\alpha_{0}}{2}\right) \sum_{\sigma \in \mathcal{E}_{h}} h_{\sigma}^{-\beta} \int_{\sigma} \llbracket \boldsymbol{v}_{h} \mathbb{1}_{\sigma}^{2} \mathrm{~d} s \\
\geq & C(\alpha)\left\|\boldsymbol{v}_{h}\right\|_{h}^{2} .
\end{aligned}
$$

Next, to ensure coercivity of the bilinear form $A_{h}(\cdot, \cdot)$, it is sufficient to choose

$$
C(\alpha)=\min \left(\frac{\alpha_{0}}{2}, \alpha_{c} v-\frac{C^{2}(1+\theta)^{2}}{2 \alpha_{0}}-\frac{\alpha_{0}}{2}\right),
$$


and $\alpha_{c}$ such that

$$
\alpha_{c} v-\frac{C^{2}(1+\theta)^{2}}{2 \alpha_{0}}-\frac{\alpha_{0}}{2}>0 .
$$

Finally, a direct application of (3.2) and (3.6) readily yields the boundedness of $A_{h}(\cdot, \cdot)$ (details can be found in e.g. [31, Lemma2.4]).

Similarly, it is possible to prove the boundedness of the bilinear form $B_{h}(\cdot, \cdot)$

$$
B_{h}\left(v_{h}, q_{h}\right) \leq C\left\|\boldsymbol{v}_{h}\right\|\left\|q_{h}\right\|_{h} \quad \forall \boldsymbol{v}_{h} \in \mathbf{V}(h), q_{h} \in L_{0}^{2}(\Omega) .
$$

Next, we employ (2.15) and (3.8) to deduce that the quadrilinear form is $\mathbf{V}(h)$-coercive with respect to the norm $\|\cdot\|_{h}$, i.e., there exists a positive constant $C$ independent of $h$ such that

$$
\mathcal{A}_{h}\left(\left(\boldsymbol{v}_{h}, q_{h}\right) ;\left(\boldsymbol{v}_{h}, q_{h}\right)\right) \geq C\left[\left\|\boldsymbol{v}_{h}\right\|_{h}^{2}+\left\|q_{h}\right\|_{h}^{2}\right], \quad \forall \boldsymbol{v}_{h} \in \mathbf{V}(h), q_{h} \in L_{0}^{2}(\Omega) .
$$

Before turning to the derivation of error estimates, we introduce the following discontinuous linear polynomial interpolation operators $\mathbf{I}_{h}: C(\Omega) \longrightarrow \mathcal{V}_{h}$ and $\Pi_{h}: L^{2}(\Omega) \longrightarrow \mathcal{Q}_{h}$ for velocity and pressure, respectively (notice that $\Pi_{h}$ is the discontinuous counterpart of the well-known Clément operator). In the next result we provide some generalities regarding such operators.

Lemma 5 (See [3,5]) For any $\boldsymbol{v} \in \mathbf{H}^{s}(\Omega)$, and $q \in H^{s-1}(\Omega)$, with $s \in\{1,2\}$, there exists a constant $C>0$ independent of $h$, such that the following approximation properties hold:

$$
\begin{aligned}
\left|\boldsymbol{v}-\mathbf{I}_{h} \boldsymbol{v}\right|_{m, K} & \leq C h^{s-m}|\boldsymbol{v}|_{s, K}, \\
\left|q-\Pi_{h} q\right|_{m, K} & \leq C h^{s-1-m}|q|_{s-1, K},
\end{aligned}
$$

where $0 \leq m \leq 1$.

Two important consequences of Lemma 5 are

$$
\begin{aligned}
\left\|\boldsymbol{v}-\mathbf{I}_{h} \boldsymbol{v}\right\| & \leq C h\|\boldsymbol{v}\|_{2, \Omega}, \\
\left\|q-\Pi_{h} q\right\|_{h} & \leq C h\|p\|_{1, \Omega} .
\end{aligned}
$$

Notice that assertion (3.10) implies the well-posedness of the discrete scheme (2.14). An application of (3.10) and Lemma 5 enables us to derive the following error estimates for velocity and pressure.

Theorem 1 (Error estimate in the mesh dependent norm) Let $\left(\boldsymbol{u}_{h}, p_{h}\right) \in \mathcal{V}_{h} \times \mathcal{Q}_{h}$ be the unique solution of (2.14) and $(\boldsymbol{u}, p)$ the unique solution of (1.4). Then, there exists $C>0$ such that

$$
\left\|\boldsymbol{u}-\boldsymbol{u}_{h}\right\|_{h}+\left\|p-p_{h}\right\|_{h} \leq C h\left(\|\boldsymbol{u}\|_{2, \Omega}+\|p\|_{1, \Omega}\right) .
$$

Proof Since $\|\boldsymbol{v}\|_{h} \leq\|\boldsymbol{v}\|$ for all $\boldsymbol{v} \in \mathbf{V}(h)$, and bounds for $\left\|\boldsymbol{u}-\mathbf{I}_{h} \boldsymbol{u}\right\|$ and $\left\|p-\Pi_{h} p\right\|_{h}$ are provided in (3.11) and (3.12), it is sufficient to estimate $\left\|\boldsymbol{u}_{h}-\mathbf{I}_{h} \boldsymbol{u}\right\|_{h}$ and $\left\|p_{h}-\Pi_{h} p\right\|_{h}$. Combining (3.10) and Lemma 3, we deduce that

$$
\begin{aligned}
\left\|\mathbf{I}_{h} \boldsymbol{u}-\boldsymbol{u}_{h}\right\|_{h}^{2}+\left\|\Pi_{h} p-p_{h}\right\|_{h}^{2} \leq & \mathcal{A}_{h}\left(\left(\mathbf{I}_{h} \boldsymbol{u}-\boldsymbol{u}_{h}, \Pi_{h} p-p_{h}\right) ;\left(\mathbf{I}_{h} \boldsymbol{u}-\boldsymbol{u}_{h}, \Pi_{h} p-p_{h}\right)\right), \\
\leq & \mathcal{A}_{h}\left(\left(\mathbf{I}_{h} \boldsymbol{u}-\boldsymbol{u}, \Pi_{h} p-p\right) ;\left(\mathbf{I}_{h} \boldsymbol{u}-\boldsymbol{u}_{h}, \Pi_{h} p-p_{h}\right)\right. \\
& +C h\left\|\mathbf{I}_{h} \boldsymbol{u}-\boldsymbol{u}_{h}\right\|_{h}\left\|\Pi_{h} p-p_{h}\right\|_{h} .
\end{aligned}
$$


To estimate the first term, we analyze all individual terms involved in the definition of $\mathcal{A}_{h}(\cdot ; \cdot)$. Using (3.7), (3.9) and Cauchy-Schwarz inequality, we see that

$$
\begin{aligned}
\left|A_{h}\left(\mathbf{I}_{h} \boldsymbol{u}-\boldsymbol{u}, \mathbf{I}_{h} \boldsymbol{u}-\boldsymbol{u}_{h}\right)\right| & \leq C\left\|\mathbf{I}_{h} \boldsymbol{u}-\boldsymbol{u}\right\|\left\|\mathbf{I}_{h} \boldsymbol{u}-\boldsymbol{u}_{h}\right\|_{h}, \\
\left|B_{h}\left(\mathbf{I}_{h} \boldsymbol{u}-\boldsymbol{u}, \Pi_{h} p-p_{h}\right)\right| & \leq C\left\|\mathbf{I}_{h} \boldsymbol{u}-\boldsymbol{u}\right\|\left\|\Pi_{h} p-p_{h}\right\|_{h}, \\
\mid d_{h}\left(\Pi_{h} p-p_{h}, \Pi_{h} p-p_{h} \mid\right. & \leq C\left\|\Pi_{h} p-p\right\|_{h}\left\|\Pi_{h} p-p_{h}\right\|_{h} .
\end{aligned}
$$

We continue in this fashion, using the definition of the bilinear form $C_{h}(\cdot, \cdot)$, Cauchy-Schwarz inequality, and Lemma 2 together with (2.8), to obtain

$$
\begin{aligned}
\left|C_{h}\left(\mathbf{I}_{h} \boldsymbol{u}-\boldsymbol{u}_{h}, \Pi_{h} p-p\right)\right| \leq & C\left[\left\|\Pi_{h} p-p\right\|_{h}\left\|\mathbf{I}_{h} \boldsymbol{u}-\boldsymbol{u}_{h}\right\|_{h}\right. \\
& \left.+h\left\|\mathbf{I}_{h} \boldsymbol{u}-\boldsymbol{u}_{h}\right\|_{h}\left(\sum_{K}\left|\Pi_{h} p-p\right|_{1, k}^{2}\right)^{1 / 2}\right],
\end{aligned}
$$

and an appeal to Lemma 5 yields

$$
\left|C_{h}\left(\mathbf{I}_{h} \boldsymbol{u}-\boldsymbol{u}_{h}, \Pi_{h} p-p\right)\right| \leq C\left[\left\|\Pi_{h} p-p\right\|_{h}\left\|\mathbf{I}_{h} \boldsymbol{u}-\boldsymbol{u}_{h}\right\|_{h}+h\left\|\mathbf{I}_{h} \boldsymbol{u}-\boldsymbol{u}_{h}\right\|_{h}\|p\|_{1, \Omega}\right] .
$$

Combining now (3.13) with (3.14), using the relation

$$
a b \leq \frac{\theta}{2} a^{2}+\frac{1}{2 \theta} b^{2}, \forall a, b \in \mathbb{R}, \theta>0,
$$

as well as (3.11), (3.12) and standard kick back arguments, we arrive at

$$
\left\|\mathbf{I}_{h} \boldsymbol{u}-\boldsymbol{u}_{h}\right\|_{h}+\left\|\Pi_{h} p-p_{h}\right\|_{h} \leq C h\left[\|\boldsymbol{u}\|_{2, \Omega}+\|p\|_{1, \Omega}\right],
$$

and the rest of the proof follows straightforwardly after applying triangle inequality.

Before proceeding to the derivation of an $L^{2}$-estimate, we define the auxiliary bilinear forms $a_{h}^{s}(\cdot, \cdot), A_{h}^{s}(\cdot, \cdot): \mathbf{V}(h) \times \mathbf{V}(h) \longrightarrow \mathbb{R}$ as follows

$$
\begin{aligned}
a_{h}^{s}(\boldsymbol{\phi}, \boldsymbol{\psi}):= & a(\boldsymbol{\phi}, \boldsymbol{\psi})-\theta \sum_{\sigma \in \mathcal{E}_{h}} v \int_{\sigma}\left(\{\boldsymbol{\varepsilon}(\boldsymbol{\phi}) \boldsymbol{n}\}_{\sigma} \cdot \llbracket \boldsymbol{\psi} \rrbracket_{\sigma}+\{\boldsymbol{\varepsilon}(\boldsymbol{\psi}) \boldsymbol{n}\}_{\sigma} \cdot \llbracket \boldsymbol{\phi} \rrbracket_{\sigma}\right) \mathrm{d} s, \\
& +\sum_{\sigma \in \mathcal{E}_{h}} \int_{\sigma} \frac{\alpha_{c} v}{h_{\sigma}^{\beta}} \llbracket \boldsymbol{\phi} \rrbracket_{\sigma} \cdot \llbracket \boldsymbol{\psi} \rrbracket_{\sigma} \mathrm{d} s, \\
A_{h}^{s}(\boldsymbol{\phi}, \boldsymbol{\psi}):= & -\sum_{K \in \mathcal{T}_{h}} \sum_{j=1}^{d+1} v \int_{s_{j+1} b_{K} s_{j}} \boldsymbol{\varepsilon}(\boldsymbol{\phi}) \boldsymbol{n} \cdot \gamma \boldsymbol{\psi} \mathrm{d} s-\theta \sum_{\sigma \in \mathcal{E}_{h}} v \int_{\sigma}\{\boldsymbol{\varepsilon}(\boldsymbol{\phi}) \boldsymbol{n}\}_{\sigma} \cdot \llbracket \gamma \boldsymbol{\psi} \rrbracket_{\sigma} \mathrm{d} s \\
& -\theta \sum_{\sigma \in \mathcal{E}_{h}} v \int_{\sigma}\{\boldsymbol{\varepsilon}(\boldsymbol{\psi}) \boldsymbol{n}\}_{\sigma} \cdot \llbracket \gamma \boldsymbol{\phi} \rrbracket_{\sigma} \mathrm{d} s+\sum_{\sigma \in \mathcal{E}_{h}} \int_{\sigma} \frac{\alpha_{c} v}{h_{\sigma}^{\beta}} \llbracket \boldsymbol{\phi} \rrbracket_{\sigma} \cdot \llbracket \boldsymbol{\psi} \rrbracket_{\sigma} \mathrm{d} s,
\end{aligned}
$$

and we notice that the bilinear form $a_{h}^{s}(\cdot, \cdot)$ is symmetric.

Theorem 2 (Error estimate in the $L^{2}$-norm) Assume that $\left(\boldsymbol{u}_{h}, p_{h}\right) \in \mathcal{V}_{h} \times \mathcal{Q}_{h}$ is the unique solution of (2.14) and $(\boldsymbol{u}, p)$ the unique solution of (1.1)-(1.3). Then, there exists $C>0$ such that

$$
\left\|\boldsymbol{u}-\boldsymbol{u}_{h}\right\|_{0, \Omega} \leq C h^{2}\left[\|\boldsymbol{u}\|_{2, \Omega}+\|p\|_{1, \Omega}+\left(\sum_{K \in \mathcal{T}_{h}}\|\boldsymbol{f}\|_{1, K}^{2}\right)^{1 / 2}\right] .
$$


Proof First, let us consider the following adjoint problem associated to (1.1)-(1.3):

Find $(\boldsymbol{\phi}, \lambda) \in \mathbf{H}_{0}^{1}(\Omega) \times L_{0}^{2}(\Omega)$ such that

$$
\begin{aligned}
-\operatorname{div}(v \boldsymbol{\varepsilon}(\boldsymbol{\phi})-\lambda \mathbf{I}) & =\boldsymbol{u}-\boldsymbol{u}_{h} & & \text { in } \Omega, \\
\operatorname{div} \boldsymbol{\phi} & =0 & & \text { in } \Omega, \\
\boldsymbol{\phi} & =\mathbf{0} & & \text { on } \Gamma,
\end{aligned}
$$

whose solutions satisfy the well-known elliptic regularity criterion [1]

$$
\|\boldsymbol{\phi}\|_{2, \Omega}+\|\lambda\|_{1, \Omega} \leq C\left\|\boldsymbol{u}-\boldsymbol{u}_{h}\right\|_{0, \Omega} .
$$

In addition, let $\phi_{I} \in \mathcal{V}_{h}$ be the usual continuous interpolant of $\phi$, for which the following approximation property holds true

$$
\left\|\boldsymbol{\phi}-\boldsymbol{\phi}_{I}\right\| \leq C h\|\boldsymbol{\phi}\|_{2, \Omega} .
$$

Multiplying (3.15) by $\boldsymbol{u}-\boldsymbol{u}_{h}$, integrating by parts, and using $\llbracket \nabla \boldsymbol{\phi} \rrbracket_{\sigma}=\mathbf{0}$, $\llbracket \lambda \rrbracket_{\sigma}=0$, we obtain

$$
\begin{aligned}
\left\|\boldsymbol{u}-\boldsymbol{u}_{h}\right\|^{2}= & a_{h}^{s}\left(\boldsymbol{u}-\boldsymbol{u}_{h}, \boldsymbol{\phi}\right)-b_{h}\left(\boldsymbol{u}-\boldsymbol{u}_{h}, \lambda\right)+(-1+\theta) \sum_{\sigma \in \mathcal{E}_{h}} v \int_{\sigma} \llbracket \boldsymbol{u}-\boldsymbol{u}_{h} \rrbracket_{\sigma} \cdot\{\boldsymbol{\varepsilon}(\boldsymbol{\phi}) \boldsymbol{n}\}_{\sigma} \mathrm{d} s \\
= & a_{h}^{s}\left(\boldsymbol{u}-\boldsymbol{u}_{h}, \boldsymbol{\phi}-\boldsymbol{\phi}_{I}\right)+a_{h}^{s}\left(\boldsymbol{u}-\boldsymbol{u}_{h}, \boldsymbol{\phi}_{I}\right)-A_{h}^{s}\left(\boldsymbol{u}-\boldsymbol{u}_{h}, \boldsymbol{\phi}_{I}\right)+A_{h}^{s}\left(\boldsymbol{u}-\boldsymbol{u}_{h}, \boldsymbol{\phi}_{I}\right) \\
& -b_{h}\left(\boldsymbol{u}-\boldsymbol{u}_{h}, \lambda-\Pi_{h} \lambda\right)-b_{h}\left(\boldsymbol{u}-\boldsymbol{u}_{h}, \Pi_{h} \lambda\right)+B_{h}\left(\boldsymbol{u}-\boldsymbol{u}_{h}, \Pi_{h} \lambda\right) \\
& -B_{h}\left(\boldsymbol{u}-\boldsymbol{u}_{h}, \Pi_{h} \lambda\right)+(-1+\theta) v \sum_{\sigma \in \mathcal{E}_{h}} \int_{\sigma} \llbracket \boldsymbol{u}-\boldsymbol{u}_{h} \rrbracket_{\sigma} \cdot\{\boldsymbol{\varepsilon}(\boldsymbol{\phi}) \boldsymbol{n}\}_{\sigma} \mathrm{d} s .
\end{aligned}
$$

Since $\phi_{I} \in \mathcal{V}_{h}$ is a continuous interpolant of $\boldsymbol{\phi}$, we note that $\boldsymbol{u}, \boldsymbol{u}_{h}, p$ and $p_{h}$ will satisfy the following problem

$$
\begin{aligned}
A_{h}^{s}\left(\boldsymbol{u}, \boldsymbol{\phi}_{I}\right)+C_{h}\left(\boldsymbol{\phi}_{I}, p\right) & =F\left(\gamma \boldsymbol{\phi}_{I}\right), \\
A_{h}^{s}\left(\boldsymbol{u}_{h}, \boldsymbol{\phi}_{I}\right)+C_{h}\left(\boldsymbol{\phi}_{I}, p_{h}\right) & =F\left(\gamma \boldsymbol{\phi}_{I}\right),
\end{aligned}
$$

and hence

$$
A_{h}^{s}\left(\boldsymbol{u}-\boldsymbol{u}_{h}, \boldsymbol{\phi}_{I}\right)=-C_{h}\left(\boldsymbol{\phi}_{h}, p-p_{h}\right) .
$$

Employing Lemma 2 together with the definition of $C_{h}$, we deduce that

$$
C_{h}\left(\phi_{h}, p-p_{h}\right)=-\left(p-p_{h}, \operatorname{div} \phi_{I}\right)+\sum_{K \in \mathcal{T}_{h}} \int_{K}\left(\boldsymbol{\phi}-\gamma \boldsymbol{\phi}_{I}\right) \nabla p \mathrm{~d} x,
$$

and combining (3.19) with (3.20) yields

$$
\begin{aligned}
\left\|\boldsymbol{u}-\boldsymbol{u}_{h}\right\|^{2}= & a_{h}^{s}\left(\boldsymbol{u}-\boldsymbol{u}_{h}, \boldsymbol{\phi}-\boldsymbol{\phi}_{I}\right) \\
& +\left[a_{h}^{s}\left(\boldsymbol{u}-\boldsymbol{u}_{h}, \boldsymbol{\phi}_{I}\right)-A_{h}^{s}\left(\boldsymbol{u}-\boldsymbol{u}_{h}, \boldsymbol{\phi}_{I}\right)+\sum_{K} \int_{K}\left(\boldsymbol{\phi}-\boldsymbol{\phi}_{I}\right) \nabla p \mathrm{~d} x\right] \\
& -\left(p-p_{h}, \boldsymbol{d i v} \boldsymbol{\phi}_{I}\right)-B_{h}\left(\boldsymbol{u}-\boldsymbol{u}_{h}, \Pi_{h} \lambda\right) \\
& +(-1+\theta) \sum_{\sigma \in \mathcal{E}_{h}} v \int_{\sigma} \llbracket \boldsymbol{u}-\boldsymbol{u}_{h} \rrbracket_{\sigma} \cdot\{\boldsymbol{\varepsilon}(\boldsymbol{\phi}) \boldsymbol{n}\}_{\sigma} \mathrm{d} s \\
& +\left[B_{h}\left(\boldsymbol{u}-\boldsymbol{u}_{h}, \Pi_{h} \lambda\right)-b_{h}\left(\boldsymbol{u}-\boldsymbol{u}_{h}, \Pi_{h} \lambda\right)\right], \\
= & T_{1}+T_{2}+T_{3}+T_{4}+T_{5}+T_{6} .
\end{aligned}
$$


Now we devote ourselves to the estimation of each term $T_{i}, i=1, \ldots, 6$. By definition of $a_{h}(\cdot, \cdot)$, relation (3.18), and the fact that $\llbracket \boldsymbol{\phi}_{I} \rrbracket_{\sigma}=\mathbf{0}$, it immediately follows that

$$
\left|T_{1}\right|=\left|a_{h}^{s}\left(\boldsymbol{u}-\boldsymbol{u}_{h}, \boldsymbol{\phi}-\boldsymbol{\phi}_{I}\right)\right| \leq C h^{2}\|\boldsymbol{u}\|_{2, \Omega}\|\boldsymbol{\phi}\|_{2, \Omega},
$$

and using (3.1), (1.1) and proceeding analogously as in the proof of [31, Lemma3.1], it is not difficult to see that

$$
\begin{aligned}
& \left|a_{h}^{s}\left(\boldsymbol{u}-\boldsymbol{u}_{h}, \boldsymbol{\phi}_{I}\right)-A_{h}^{s}\left(\boldsymbol{u}-\boldsymbol{u}_{h}, \boldsymbol{\phi}_{I}\right)+\sum_{K \in \mathcal{T}_{h}} \int_{K}\left(\boldsymbol{\phi}-\gamma \boldsymbol{\phi}_{I}\right) \nabla p \mathrm{~d} x\right| \\
& \leq C h^{2}\left[\|\boldsymbol{u}\|_{2, \Omega}+\left(\sum_{K \in \mathcal{T}_{h}}\|\boldsymbol{f}\|_{1, K}^{2}\right)^{1 / 2}\right]\left\|\boldsymbol{\phi}_{I}\right\|_{1, \Omega} .
\end{aligned}
$$

Therefore we can apply (3.18) to get $\left\|\phi_{I}\right\|_{1} \leq C\|\boldsymbol{\phi}\|_{2}$, and consequently we have the following bound for $T_{2}$

$$
\left|T_{2}\right| \leq C h^{2}\left[\|\boldsymbol{u}\|_{2, \Omega}+\left(\sum_{K \in \mathcal{T}_{h}}\|\boldsymbol{f}\|_{1, K}^{2}\right)^{1 / 2}\right]\|\boldsymbol{\phi}\|_{2, \Omega} .
$$

To deal with $T_{3}$, it is enough to apply (3.16), (3.18) and Theorem 1 to have

$$
\left|T_{3}\right|=\left|\left(p-p_{h}, \operatorname{div} \phi_{I}\right)\right|=\mid\left(p-p_{h}, \operatorname{div}\left(\boldsymbol{\phi}-\boldsymbol{\phi}_{I}\right) \mid \leq C h^{2}\|p\|_{1, \Omega}\|\boldsymbol{\phi}\|_{2, \Omega} .\right.
$$

In order to bound $T_{4}$, we first we note that

$$
B\left(\boldsymbol{u}, \Pi_{h} \lambda\right)-B_{h}\left(\boldsymbol{u}_{h}, \Pi_{h} \lambda\right)=G_{h}\left(\Pi_{h} \lambda\right)+d_{h}\left(p, \Pi_{h} \lambda\right)-d_{h}\left(p-p_{h}, \Pi_{h} \lambda\right),
$$

and then observe that Cauchy-Schwarz inequality together with Theorem 1 and the assumption that the operator $\Pi_{h}$ is $H^{1}$-stable and all stabilization parameters $\alpha_{i}$ are bounded, gives

$$
\begin{aligned}
\left|G_{h}\left(\Pi_{h} \lambda\right)\right| & \leq C h^{2}\|\boldsymbol{f}\|_{0, \Omega}\|\lambda\|_{1, \Omega}, \\
\left|d_{h}\left(p-p_{h}, \Pi_{h} \lambda\right)\right| & \leq\left[d_{h}\left(p-p_{h}, p-p_{h}\right)\right]^{1 / 2}\left[d_{h}\left(\Pi_{h} \lambda, \Pi_{h} \lambda\right)\right]^{1 / 2}, \\
& \leq C\left\|p-p_{h}\right\|_{h} h\|\lambda\|_{1, \Omega}, \\
& \leq C h^{2}\|p\|_{1, \Omega}\|\lambda\|_{1, \Omega},
\end{aligned}
$$

and in the same manner we obtain

$$
\left|d_{h}\left(p, \Pi_{h} \lambda\right)\right| \leq C h^{2}\|p\|_{1, \Omega}\|\lambda\|_{1, \Omega} .
$$

Substituting these estimates in (3.21), we can assert that

$$
\left|T_{4}\right|=\left|B_{h}\left(\boldsymbol{u}-\boldsymbol{u}_{h}, \Pi_{h} \lambda\right)\right| \leq C h^{2}\left(\|\boldsymbol{f}\|_{0, \Omega}+\|p\|_{1, \Omega}\right)\|\lambda\|_{1, \Omega} .
$$


To bound $T_{5}$, we employ again Cauchy-Schwarz inequality, trace inequality (2.4), Theorem 1 , and the definition of $\|\cdot\|_{h}$ with $\beta=3$ to obtain

$$
\begin{aligned}
\left|T_{5}\right| & =\left|(-1+\theta) \sum_{\sigma \in \mathcal{E}_{h}} v \int_{\sigma} \llbracket \boldsymbol{u}-\boldsymbol{u}_{h} \rrbracket_{\sigma} \cdot\{\boldsymbol{\varepsilon}(\boldsymbol{\phi}) \boldsymbol{n}\}_{\sigma} \mathrm{d} s\right| \\
& \leq\left(\sum_{\sigma \in \mathcal{E}_{h}} \int_{\sigma} h_{\sigma}^{-\beta} \llbracket \boldsymbol{u}-\boldsymbol{u}_{h} \rrbracket_{\sigma}^{2} \mathrm{~d} s\right)^{1 / 2} \times\left(\sum_{\sigma \in \mathcal{E}_{h}} \int_{\sigma} h_{\sigma}^{\beta}\{\boldsymbol{\varepsilon}(\boldsymbol{\phi}) \boldsymbol{n}\}_{\sigma}^{2} \mathrm{~d} s\right), \\
& \leq C h\left\|\boldsymbol{u}-\boldsymbol{u}_{h}\right\|_{h}\|\boldsymbol{\phi}\|_{2, \Omega}, \\
& \leq C h^{2}\|\boldsymbol{u}\|_{2, \Omega}\|\boldsymbol{\phi}\|_{2, \Omega} .
\end{aligned}
$$

Proceeding in a similar way and using the $H^{1}$-stability of $\Pi_{h}$, we find that

$$
\left|\sum_{\sigma \in \mathcal{E}_{h}} \int_{\sigma} \llbracket \boldsymbol{u}-\boldsymbol{u}_{h} \rrbracket_{\sigma}\left\{\Pi_{h} \lambda\right\}_{\sigma} \mathrm{d} s\right| \leq C h^{2}\|\boldsymbol{u}\|_{2, \Omega}\|\lambda\|_{1, \Omega},
$$

and

$$
\left|\sum_{\sigma \in \mathcal{E}_{h}} \int_{\sigma} \llbracket \gamma\left(\boldsymbol{u}-\boldsymbol{u}_{h}\right) \rrbracket_{\sigma}\left\{\Pi_{h} \lambda\right\}_{\sigma} \mathrm{d} s\right| \leq C h^{2}\|\boldsymbol{u}\|_{2, \Omega}\|\lambda\|_{1, \Omega} .
$$

Therefore, using the definitions of $b_{h}(\cdot, \cdot)$ and $B_{h}(\cdot, \cdot)$, the following bound for $T_{6}$ readily follows

$$
\left|T_{6}\right|=\left|B_{h}\left(\boldsymbol{u}-\boldsymbol{u}_{h}, \Pi_{h} \lambda\right)-b_{h}\left(\boldsymbol{u}-\boldsymbol{u}_{h}, \Pi_{h} \lambda\right)\right| \leq C h^{2}\|\boldsymbol{u}\|_{2, \Omega}\|\lambda\|_{1, \Omega} .
$$

Collecting the derived estimates for $T_{i}, i=1, \ldots, 6$ all together in (2.16), using the regularity result (3.17), and dividing by $\left\|\boldsymbol{u}-\boldsymbol{u}_{h}\right\|_{0, \Omega}$, we complete the proof.

Remark 2 We stress that the analysis presented here can be extended straightforwardly to the case of Stokes formulations with non-homogeneous boundary conditions.

\section{Numerical Examples}

We now present some numerical examples using the proposed DFVE method described in Sect. 2, which confirm the theoretical results of Sect. 3. A conjugate gradient method with lumped pressure mass preconditioning (see e.g. [40]) is employed to solve the linear system arising from the discrete formulation.

\subsection{Example 1: Experimental Convergence on the Unit Square}

First we assess the accuracy of the method by comparing the approximate and the exact solutions of (1.1)-(1.2) given by the smooth functions

$$
\boldsymbol{u}(x, y)=\left(\begin{array}{c}
-256 x^{2}(x-1)^{2} y(y-1)(2 y-1) \\
256 y^{2}(y-1)^{2} x(x-1)(2 x-1)
\end{array}\right), \quad p(x, y)=(x-1 / 2)(y-1 / 2),
$$

defined on the unit square $\Omega=(0,1)^{2}$ and with $v=1$ (see [7]). We construct a family of uniform triangular meshes for $\Omega$ with $N_{h_{i}}=\left(5 \cdot 2^{i}+1\right)^{2}$ vertices, $i=0, \ldots, 7$, where 
Table 1 Experimental convergence histories for the stabilized DFVE approximation of the Stokes problem $(1.1)-(1.3)$

\begin{tabular}{llllllll}
\hline$N_{h}$ & $h$ & $e_{0}(\boldsymbol{u})$ & $r_{0}(\boldsymbol{u})$ & $e_{h}(\boldsymbol{u})$ & $r_{h}(\boldsymbol{u})$ & $e_{h}(p)$ & $r_{h}(p)$ \\
\hline Example 1 & & & & & & & \\
36 & 0.2828430 & 0.4476130 & - & 3.824174 & - & 0.591913 & - \\
121 & 0.1414215 & 0.1223365 & 1.87141 & 2.008662 & 0.928916 & 0.197659 & 1.58237 \\
441 & 0.0707107 & 0.0286545 & 2.09402 & 1.015231 & 0.984427 & 0.082143 & 1.26681 \\
1,681 & 0.0353553 & 0.0068536 & 2.06384 & 0.508904 & 0.996337 & 0.040325 & 1.03077 \\
6,561 & 0.0176777 & 0.0015946 & 1.99323 & 0.254637 & 0.998954 & 0.020103 & 1.00654 \\
25,921 & 0.0088388 & 0.0003965 & 1.99937 & 0.127375 & 0.999361 & 0.010022 & 1.00098 \\
103,041 & 0.0044194 & 0.0000986 & 2.00012 & 0.061792 & 1.002480 & 0.006419 & 1.00107 \\
410,881 & 0.0022981 & 0.0000242 & 1.99981 & 0.031048 & 1.000172 & 0.003574 & 1.03420 \\
795,684 & 0.0011653 & 0.0000123 & 1.99992 & 0.015039 & 1.000025 & 0.001738 & 1.00152 \\
Example 2 & & & & & & & \\
8 & $0.6667 \mathrm{e}-01$ & $4.3908 \mathrm{e}-01$ & - & 4.193831 & - & 1.897451 & - \\
512 & $4.8621 \mathrm{e}-04$ & $3.6750 \mathrm{e}-04$ & 1.439959 & 0.143281 & 0.896297 & 0.019056 & 1.006706 \\
1,331 & $1.6732 \mathrm{e}-04$ & $7.4219 \mathrm{e}-05$ & 1.499691 & 0.071263 & 0.964722 & 0.007378 & 0.966579 \\
2,744 & $7.5861 \mathrm{e}-05$ & $2.0427 \mathrm{e}-05$ & 1.631009 & 0.042446 & 0.969506 & 0.003783 & 0.974878 \\
4,913 & $4.0690 \mathrm{e}-05$ & $7.2351 \mathrm{e}-06$ & 1.662721 & 0.028118 & 0.936125 & 0.002274 & 0.987362 \\
8,000 & $2.4299 \mathrm{e}-05$ & $2.7017 \mathrm{e}-06$ & 1.910762 & 0.019980 & 0.967367 & 0.001412 & 0.984081 \\
12,167 & $1.5652 \mathrm{e}-05$ & $1.1388 \mathrm{e}-06$ & 1.963423 & 0.013781 & 0.985478 & 0.000973 & 0.986609 \\
17,576 & $6.8721 \mathrm{e}-06$ & $6.1141 \mathrm{e}-07$ & 1.982419 & 0.007632 & 0.989601 & 0.000621 & 0.990167 \\
24,678 & $2.9874 \mathrm{e}-06$ & $2.5177 \mathrm{e}-07$ & 1.993540 & 0.003782 & 0.993177 & 0.000419 & 0.995237 \\
32,972 & $1.7598 \mathrm{e}-06$ & $1.3278 \mathrm{e}-07$ & 1.995862 & 0.002105 & 0.997420 & 0.000287 & 0.995899 \\
\hline
\end{tabular}

Here we have considered the parameters $\theta=-1, \beta=1, \alpha_{c}=100, \alpha_{d}=0.05, \alpha_{e}=0.1$ for Example 1, whereas we set $\alpha_{c}=5,000$ and $\alpha_{d}=0.001$ for Example 2

the convergence of the approximate solutions is measured by errors in different norms and corresponding observed rates defined as

$$
\begin{aligned}
& e_{h}(\boldsymbol{u}):=\left\|\boldsymbol{u}-\boldsymbol{u}_{h}\right\|_{h}, e_{0}(\boldsymbol{u}):=\left\|\boldsymbol{u}-\boldsymbol{u}_{h}\right\|_{0, \Omega}, e_{h}(p):=\left\|p-p_{h}\right\|_{h}, \\
& r_{h}(\boldsymbol{u}):=\frac{\log \left(e_{h}(\boldsymbol{u}) / \hat{e}_{h}(\boldsymbol{u})\right)}{\log (h / \hat{h})}, r_{0}(\boldsymbol{u}):=\frac{\log \left(e_{0}(\boldsymbol{u}) / \hat{e}_{0}(\boldsymbol{u})\right)}{\log (h / \hat{h})}, r_{h}(p):=\frac{\log \left(e_{h}(p) / \hat{e}_{h}(p)\right)}{\log (h / \hat{h})} .
\end{aligned}
$$

Here $e$ and $\hat{e}$ denote errors computed on two consecutive meshes of sizes $h$ and $\hat{h}$. These quantities are depicted in Table 1 (top rows). Here the stabilization parameters assume the values $\beta=1, \alpha_{c}=100, \alpha_{d}=0.05, \alpha_{e}=0.1$. An experimental quadratic convergence rate is observed for the velocity in the $L^{2}$-norm, whereas pressure and velocity in the energy norm exhibit a convergence rate of order $h$. These results agree with the theoretical error estimates from Sect. 3. Approximate discontinuous solutions are presented in Fig. 3.

Remark 3 In addition, we have tested the accuracy of the method depending on the symmetry of the formulation (encoded in the parameter $\theta$ ) and on the stabilization parameters $\alpha_{c}, \beta$. We consider eight scenarios where we choose $\alpha_{c} \in\{0.1,1,000\}, \theta \in\{1,-1\}$ and $\beta \in\{1,3\}$. The error histories are presented in Fig. 4, where we observe that the expected orders of convergence are attained for all cases except for $\left(\alpha_{c}=0.1, \theta=-1, \beta=1\right)$ and $\left(\alpha_{c}=\right.$ 


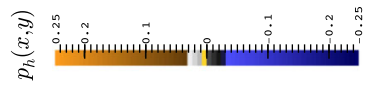
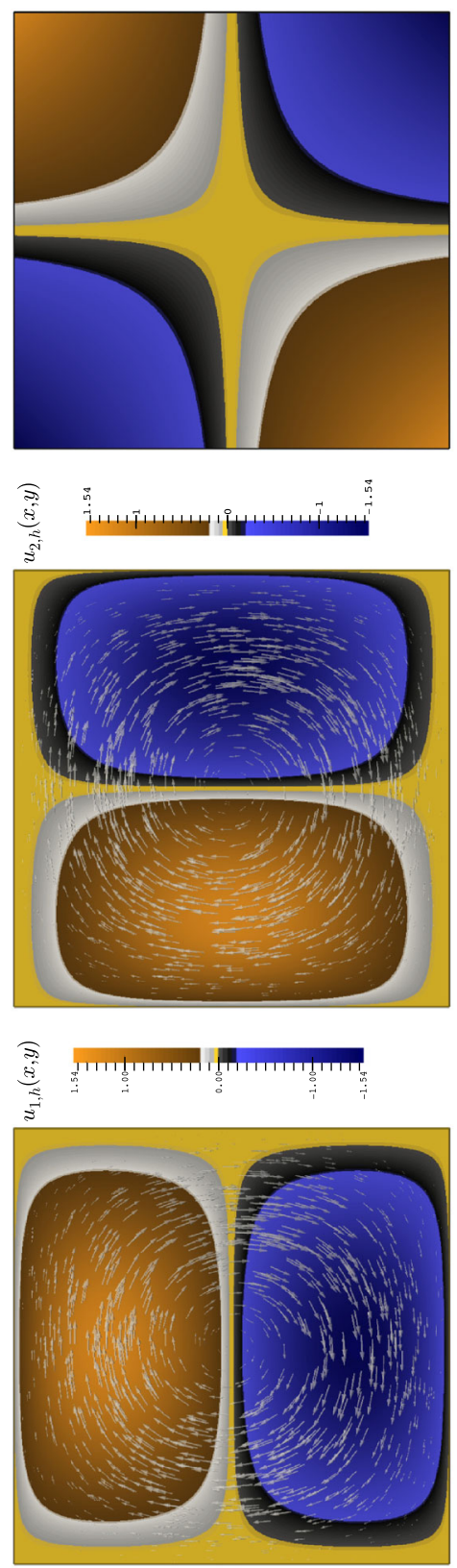
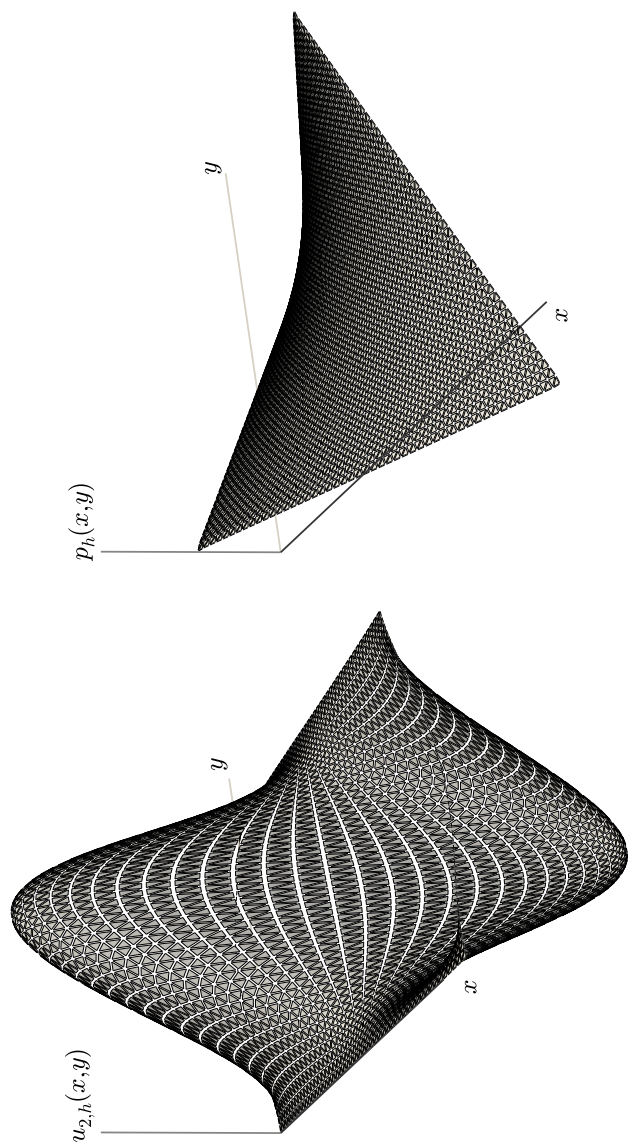

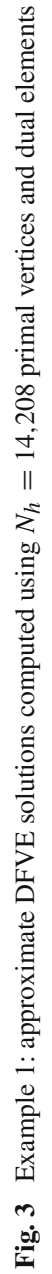



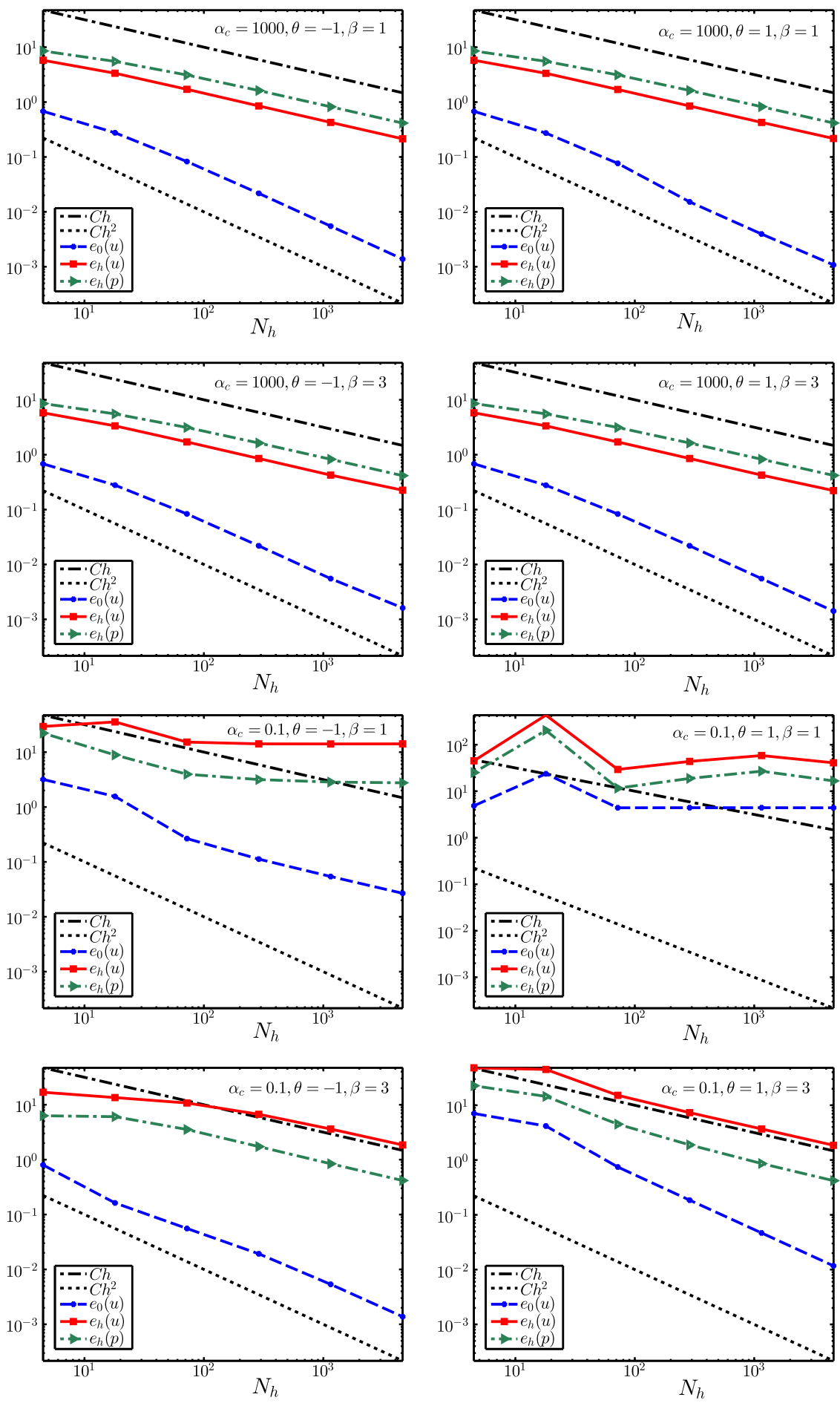

Fig. 4 Example 1: observed convergence rates for velocity in the $L^{2}$ - and mesh-dependent norms and pressure, for different parameter values $\alpha_{c} \in\{0.1,1,000\}, \theta \in\{1,-1\}$ and $\beta \in\{1,3\}$ 

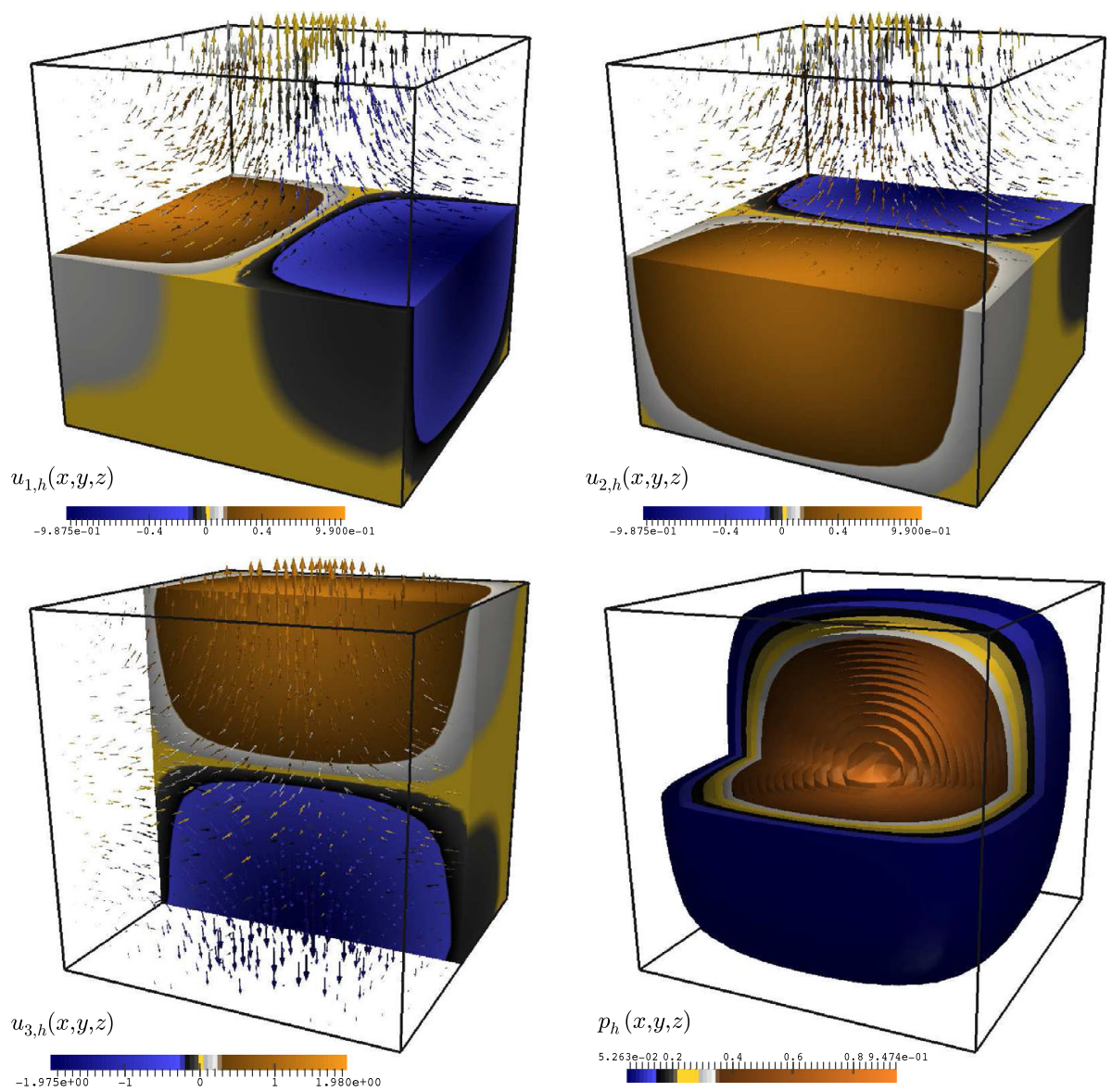

Fig. 5 Example 2: approximate DFVE velocity components and pressure contours for the accuracy test on a cube

$0.1, \theta=1, \beta=1)$. These results, along with those presented in the precedent paragraph, suggest that for $\beta=1$ the stabilization parameter $\alpha_{c}$ needs to be large, whereas either small or large values of $\alpha_{c}$ are sufficient to achieve the expected convergence for $\beta=3$. We can conclude that the theoretical requirements on the stabilization parameters $\left(\alpha_{c}, \alpha_{d}, \alpha_{e}\right.$ and $\left.\beta\right)$ are consistently met in the performed numerical results.

\subsection{Example 2: Experimental Convergence on a Cube}

We also test the 3D implementation by analyzing the convergence of the DFVE approximations to the following exact solutions of (1.1)-(1.2)

$$
\boldsymbol{u}(x, y, z)=\left(\begin{array}{c}
\cos (\pi x) \sin (\pi y) \sin (\pi z) \\
\sin (\pi x) \cos (\pi y) \sin (\pi z) \\
-2 \sin (\pi x) \sin (\pi y) \cos (\pi z)
\end{array}\right), \quad p(x, y, z)=\sin (\pi x) \sin (\pi y) \sin (\pi z),
$$

defined on the unit cube $\Omega=(0,1)^{3}$. We set $v=1$ and forcing and boundary terms are chosen according to these solutions. The approximate fields are presented in Fig. 5. As in 

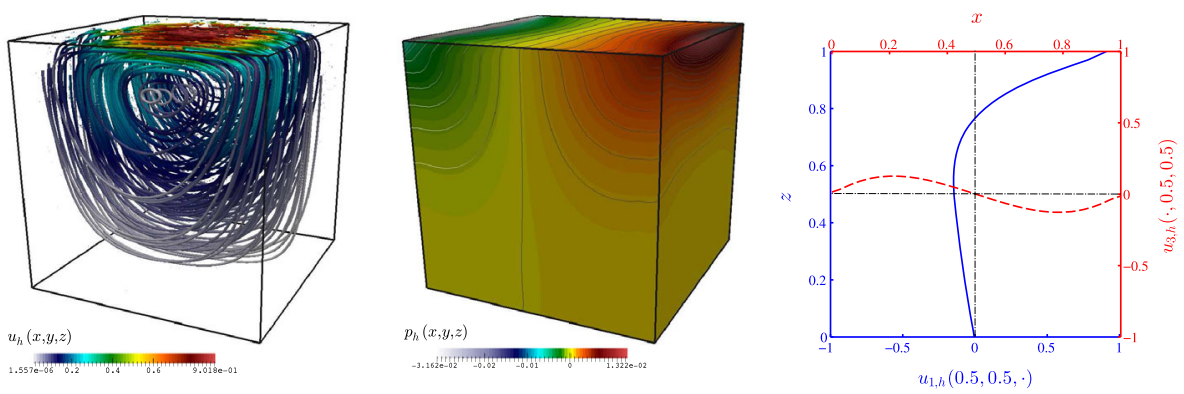

Fig. 6 Example 3: approximate velocity streamlines (left), pressure distribution (center) and velocity profiles $(r i g h t)$ for the lid-driven cavity test employing the DFVE method

the previous example, we take $\theta=-1, \beta=1, \alpha_{e}=0.1$, but modify $\alpha_{c}=5,000$ and $\alpha_{d}=0.001$. A family of tetrahedral meshes for $\Omega$ is constructed having $N_{h_{i}}=(2+3 i)^{3}$ vertices, with $i=0, \ldots, 7$. The expected rates of convergence are observed for both velocity and pressure in all norms (see the bottom rows in Table 1).

\subsection{Example 3: The Lid-Driven Cavity}

Finally we perform the lid-driven cavity benchmark, where the flow in a closed box is driven by the uniform motion of the top lid. The domain is again the unit cube $\Omega=(0,1)^{3}$ and the primal unstructured mesh consists of 29,791 nodes and 172,800 tetrahedral elements. The viscosity is set to $v=0.01$, the body force is $\boldsymbol{f}=\mathbf{0}$ and we impose no-slip boundary conditions on the walls and bottom lids of the cube, whereas on the top lid we fix the smooth velocity profile $\boldsymbol{u}=(16 x(1-x) y(1-y), 0,0)^{\mathrm{t}}$ (which ensures regularity of the discrete solution, see e.g. [38]). Approximate solutions are presented in Fig. 6, where we observe well resolved velocity patterns and mid-line profiles, along with stable pressure near the top lid. These results agree with those reported in e.g. [38].

\section{Concluding Remarks}

In this paper we proposed an analyzed the convergence of novel equal-order discontinuous finite volume element methods for the Stokes equations. Several numerical tests in twoand three spatial dimensions exhibit either linear, or near linear convergence for velocity and pressure in the mesh-dependent norms, which also match the theoretical rates of convergence predicted by our analysis.

Further directions for development include the statement and analysis of fully DFVE methods for coupled flow and transport problems with applications in porous media [30] and sedimentation-consolidation processes [11], and generalization of DFVE methods for fully mixed formulations in flow problems and elasticity. We are also interested in the derivation of a posteriori error estimates [13], the superconvergence of approximate solutions via local postprocessing [29,39] and on vorticity-based formulations [2,24]. Some of these topics will be covered in a forthcoming contribution.

Acknowledgments We thank Dr. Thirupathi Gudi (IISc, Bangalore) for his valuable suggestions during the early stage of this work, and we gratefully acknowledge the support by the University of Lausanne. 


\section{References}

1. Agmon, S.: Lectures on Elliptic Boundary Value Problems. AMS Providence, Rhode Island (2010)

2. Anaya, V., Mora, D., Ruiz-Baier, R.: An augmented mixed finite element method for the vorticityvelocity-pressure formulation of the Stokes equations. Comput. Methods Appl. Mech. Eng. 267, 261-274 (2013)

3. Arnold, D.N., Brezzi, F., Cockburn, B., Marini, L.D.: Unified analysis of discontinuous Galerkin methods for elliptic problems. SIAM J. Numer. Anal. 39, 1749-1779 (2002)

4. Badia, S., Codina, R., Gudi, T., Guzmán, J.: Error analysis of discontinuous Galerkin methods for the Stokes problem under minimal regularity. IMA J. Numer. Anal. (2013). doi:10.1093/imanum/drt023

5. Baroli, D., Quarteroni, A., Ruiz-Baier, R.: Convergence of a stabilized discontinuous Galerkin method for incompressible nonlinear elasticity. Adv. Comput. Math. 39, 425-443 (2013)

6. Becker, R., Capatina, D., Joie, J.: Connections between discontinuous Galerkin and nonconforming finite element methods for the Stokes equations. Numer. Methods Partial Differ. Equ. 28, 1013-1041 (2012)

7. Bercovier, M., Engelman, M.: A finite element for the numerical solution of viscous incompressible flows. J. Comput. Phys. 30, 181-201 (1979)

8. Berggren, M., Ekström, S.E., Nordström, J.: A discontinuous Galerkin extension of the vertex-centered edge-based finite volume method. Commun. Comput. Phys. 5, 456-468 (2009)

9. Bi, C., Geng, J.: A discontinuous finite volume element method for second-order elliptic problems. Numer. Methods Partial Differ. Equ. 28, 425-440 (2012)

10. Brezzi, F., Fortin, M.: Mixed and Hybrid Finite Element Methods, vol. 15 of Springer Series in Computational Mathematics. Springer, New York (1991)

11. Bürger, R., Ruiz-Baier, R., Torres, H.: A stabilized finite volume element formulation for sedimentationconsolidation processes. SIAM J. Sci. Comput. 34, B265-B289 (2012)

12. Cai, Z.: On the finite volume element method. Numer. Math. 58, 713-735 (1991)

13. Chatzipantelidis, P., Makridakis, C., Plexousakis, M.: A-posteriori error estimates for a finite volume method for the Stokes problem in two dimensions. Appl. Numer. Math. 46, 45-58 (2003)

14. Chou, S.C.: Analysis and convergence of a covolume method for the generalized Stokes problem. Math. Comput. 66, 85-104 (1997)

15. Chung, E.T., Kim, H.H., Widlund, O.B.: Two-level overlapping Schwarz algorithms for a staggered discontinuous Galerkin method. SIAM J. Numer. Anal. 51(1), 47-67 (2013)

16. Cockburn, B., Kanschat, G., Schötzau, D.: An equal-order DG method for the incompressible NavierStokes equations. J. Sci. Comput. 40, 188-210 (2009)

17. Cockburn, B., Kanschat, G., Schötzau, D., Schwab, C.: Local discontinuous Galerkin methods for the Stokes system. SIAM J. Numer. Anal. 40, 319-343 (2002)

18. Cui, M., Ye, X.: Superconvergence of finite volume methods for the Stokes equations. Numer. Methods PDEs 25, 1212-1230 (2009)

19. Cui, M., Ye, X.: Unified analysis of finite volume methods for the Stokes equations. SIAM J. Numer. Anal. 48, 824-839 (2010)

20. Ewing, R.E., Lin, T., Lin, Y.: On the accuracy of the finite volume element method based on piecewise linear polynomials. SIAM J. Numer. Anal. 39, 1865-1888 (2002)

21. Feistauer, M., Felcman, J., Lukáčová-Medvid'ová, M.: Combined finite element-finite volume solution of compressible flow. J. Comput. Appl. Math. 63, 179-199 (1995)

22. Fortin, M.: Finite element solution of the Navier-Stokes equations. Acta Numer. 5, 239-284 (1993)

23. Ganesan, S., Matthies, G., Tobiska, L.: Local projection stabilization of equal order interpolation applied to the Stokes problem. Math. Comput. 77, 2039-2060 (2008)

24. Gatica, G.N., Márquez, A., Sánchez, M.A.: Analysis of a velocity-pressure-pseudostress formulation for the stationary Stokes equations. Comput. Methods Appl. Mech. Eng. 199, 1064-1079 (2010)

25. Girault, V., Rivière, B., Wheeler, M.F.: A discontinuous Galerkin method with nonoverlapping domain decomposition for the Stokes and Navier-Stokes problems. Math. Comput. 74, 53-84 (2005)

26. Girault, V., Wheeler, M.F.: Discontinuous Galerkin methods. In: Partial Differential Equations, Computational Methods in Applied Sciences, vol. 16, pp. 3-26 (2008)

27. Hansbo, P., Larson, M.G.: Discontinuous Galerkin methods for incompressible and nearly incompressible elasticity by Nitsche's method. Comput. Methods Appl. Mech. Eng. 191, 1895-1908 (2002)

28. Hansbo, P., Larson, M.G.: Piecewise divergence-free discontinuous Galerkin methods for Stokes flow. Commun. Numer. Meth. Eng. 24, 355-366 (2008)

29. Kim, H.H., Chung, E.T., Lee, C.S.: A staggered discontinuous Galerkin method for the Stokes system. SIAM J. Numer. Anal. 51, 3327-3350 (2013)

30. Kumar, S.: A mixed and discontinuous Galerkin finite volume element method for incompressible miscible displacement problems in porous media. Numer. Methods Partial Differ. Equ. 28, 1354-1381 (2012) 
31. Kumar, S., Nataraj, N., Pani, A.K.: Discontinuous Galerkin finite volume element methods for second order linear elliptic problems. Numer. Meth. Partial Differ. Equ. 25, 1402-1424 (2009)

32. Lazarov, R., Ye, X.: Stabilized discontinuous finite element approximations for Stokes equations. J. Comput. Appl. Math. 198, 236-252 (2007)

33. Li, J., Chen, Z.: A new stabilized finite volume method for the stationary Stokes equations. Adv. Comput. Math. 30, 141-152 (2009)

34. Lin, Y., Liu, J., Yang, M.: Finite volume element methods: an overview of recent developments. Int. J. Numer. Anal. Model. B 4, 14-24 (2013)

35. Liu, J., Mu, L., Ye, X.: An adaptive discontinuous finite volume element method for elliptic problems. J. Comput. Appl. Math. 235, 5422-5431 (2011)

36. Liu, J., Mu, L., Ye, X., Jari, R.: Convergence of the discontinuous finite volume method for elliptic problems with minimal regularity. J. Comput. Appl. Math. 236, 4537-4546 (2012)

37. Nicaise, S., Djadel, K.: Convergence analysis of a finite volume method for the Stokes system using non-conforming arguments. IMA J. Numer. Anal. 25, 523-548 (2005)

38. Notsu, H.: Numerical computations of cavity flow problems by a pressure stabilized characteristic-curve finite element scheme. Trans. Jpn. Soc. Comput. Eng. Sci. 08, 20080032-20080051 (2008)

39. Quarteroni, A., Ruiz-Baier, R.: Analysis of a finite volume element method for the Stokes problem. Numer. Math. 118, 737-764 (2011)

40. Quarteroni, A., Valli, A.: Numerical Approximation of Partial Differential Equations. Springer, Berlin (1997)

41. Ruiz-Baier, R., Torres, H.: Numerical solution of a multidimensional sedimentation problem using finite volume-elements. Appl. Numer. Math. (2014). doi:10.1016/j.apnum.2013.12.006

42. Schötzau, D., Schwab, C., Toselli, A.: Mixed hp-DGFEM for incompressible flows. II. Geometric edge meshes. IMA J. Numer. Anal. 24, 273-308 (2004)

43. Shahbazi, K., Fischer, P.F., Ethier, C.R.: A high-order discontinuous Galerkin method for the unsteady incompressible NavierStokes equations. J. Comput. Phys. 222, 391-407 (2007)

44. Toselli, A.: Hp discontinuous Galerkin approximations for the Stokes problem. Math. Models Methods Appl. Sci. 12, 1565-1597 (2002)

45. Yang, Q., Jiang, Z.: A discontinuous mixed covolume method for elliptic problems. J. Comput. Appl. Math. 235, 2467-2476 (2011)

46. Ye, X.: On the relationship between finite volume and finite element methods applied to the Stokes equations. Numer. Methods Partial Differ. Equ. 17, 440-453 (2001)

47. Ye, X.: A new discontinuous finite volume method for elliptic problems. SIAM J. Numer. Anal. 42, 1062-1072 (2004)

48. Ye, X.: A discontinuous finite volume method for the Stokes problem. SIAM J. Numer. Anal. 44, 183-198 (2006)

49. Yin, Z., Jiang, Z., Xu, Q.: A discontinuous finite volume method for the Darcy-Stokes equations. J. Appl. Math. 2012, 761242-761258 (2012) 